\title{
COLLISIONLESS ABSORPTION AND \\ EMISSION OF ELECTROMAGNETIC WAVES \\ BY A BOUNDED PLASMA
}

Thesis by

Frederick Lee Hinton

In Partial Fulfillment of the Requirements

For the Degree of

Doctor of Philosophy

\author{
California Institute of Technology \\ Pasadena, Califorria
}

1967

(Submitted May 15, 1967) 
The author wishes to express his appreciation to Professor R. W. Gould for his guidance and encouragement during this investigation and for the numericai calculations on which Figure 2 is based. Thanks are due also to Mrs. Ruth Stratton for her expert preparation of the manuscript and for secretarial assistance throughout the investigation.

The autinor acknowledges support by the National Science Foundation through a Graduate Fellowship, and by the United States Air Force Office of Scientific Research under Contract $\operatorname{Ar} 49(638)-1462$. 


\section{ABSTRACT}

The absorption and emission of electromagnetic waves by a hot bounded plasma can be greatly influenced by the collective behavior of the plasma. A homogeneous slab model of a hot plasma is used in a theorctical otudy of thcso colicctive cffceto. $\Lambda$ boundary-value problem is solved to find the fields in the plasma when a plane wave is incident on the plasma boundary. Power is found to be absorbed from the incident wave because of the Landau damping of longitudinal plasma waves excited in the plasma. If the incident wave frequency is close to one of the plasma wave resonance frequencies, the absorption may be suhstantial, but orly if the tandan àmping rate is approximateiy equal to the radiation amping rate, i.e. if the plasma is "matched" to the radiation field. The width of an absorption line is the sum of the Landau damping and radiation damping widths. Electromagnetic noise radiation is emitted from a collisionless plasma because of the coupling of the field fluctuations in the plasma to the external radiation field at the plasma boundary. Plasma wave fluctuations are excited by fast electrons in the plasma and are reinforced by the collective response of the plasma at the resonance frequencies. Emission and absorption are related by Kirchhoff's law for a thermal plasma. For a nonthermal plasma the emission may be enhanced if there are many electrons in a high-energy tail of the distribution function. 


$$
-i v-
$$

CONTENTS

I. INTRODUCTION

II. SELF-CONSISTENT SOLUTION OF MAXWELL'S EQUATIONS AND IHE PARTICLE EQUATIONS OF MOTION

A. Fourier Analysis of Maxwell's Equations 8

B. Perturbation Analysis of the Particle Equations of Motion

c. Hourier Coefriclents of the Currenl Density

D. Self-Consistent Solution of the Boundary Value rroblem

B. The Absorption Coefficient of a Collisionless Plasma Slab

C. Absorption Lines ard Scattering Resonances

TV. COLLISIONLESS NOISE RADIATION FROM A PLASMA SLAB

A. Surface Emissivity 56

B. Electric Field Fluctuations in a Plasma Slab 62

C. Enhancement of Emission Lines in Non-Thermal
Plasmas

D. The Affect of Collisions on "Collisionless" Absorption and Emission

V. SUMMARY AND CONCLUSIONS 80

$\begin{array}{ll}\text { REFERENCES . } & 86\end{array}$ 


\section{INTRODUCTION}

A plasma is a quasi-neutral collection of electrically chargea particles. As such, it interacts strongly with electromagnetic fields. By observing the interaction of a plasma with electromagnetic waves, it is possiole to learn something about the behavior of this collection of particles. Of particular interest in this investigation is the collective behavior, in winich large numbers of particles participate, which results in the propagation of longitudinal waves with frequencies close to the plasma frequency $\omega_{p}$. These waves may be coupled to the electromagnetic waves if the plasma has boundaries. The existence of such Iongitudinal plasma waves may be manifested in peaks in the amount of energy absorbeó from an incident electromagnetic wave, as a function of frequency. At these resonance frequencies, the amount of energy scattered, or reflected, by the plasma may also have maxima.

These properties have been observed experimentally in gaseous discharge plasmas (1). The positions of the resonance frequencies have been calculated, in good agreement with experiment, by Parker, Nickel, and Gould (2), but the mechanism of power absorption has not been fully explained. Also observed, in microwave experiments by Kerzar and Weissglas (3), and by Gould (4), are similar peaks in the noise emission, which are not completely understood.

The purpose of this investigation is to show how the power absorption at the resonance frequencies of a bounded plasma is reiated to Landau damping (5), and to discuss the corresponding noise emission mechanism. Expressions for the absorption coefficient and the emissivity are derived theoretically for a bounded plasma which is not 
necessarily in thermal equilibrium. In the special case of thermal equilibrium the expression for the emissivity agrees with the result obtained from Kirchhoff's law using the expression for the absorption coefficient.

The type of plasma which is of most interest here is the nearly collisionless hot plasma. The mean kinetic energy of the particles is assumed to be much larger than their mean potential energy due to the Couiomb forces between them. In a thermal equilibrium plasma this may be expressed as

$$
\frac{x^{m}}{n^{1 / 3} e^{2}} \equiv \Lambda^{2 / 3}>1
$$

which is satisfiea in most cases of interest. The basis of the theoretical description is then the quasi-free motions of the charged particles in the macroscopic electric field of the plasma, with particle encounters treated as small perturbations. This contrasts with the fluid description which is often used, and which is usually justified (in the absence of a strong magnetic field) oniy if collisions are very frequent $(6)$.

We are concerned here with observable quantities, which might be measured in a laboratory experiment. Since the plasma must be produced in the laporatory, it is necessarily bounded in spatial extent. This feature is quite important for the electromagnetic properties of the plasma, but only a few problems involving hot bounded plasmas have been solved. Most of the theoretical work on hot plasmas is concermed with the case where there are no boundaries (infinite plasma) or where boundary effects are not of primary importance. One problem which has 
been solved, however, and which is closely related to the proilems considered in this investigation, is the "plasma capacitor" problem ( 7 ). A plasma of uniform density is contained between the plates of an infinite plane capacitor, and the particles of the plasma are supposed to undergo specular reflection at the plates. The problem is to fino the specific impedance of the capacitor, i.e., the voltage drop across the plates divided by the dispiacement current per unit area as a function of frequency. The solution shows that there are resonances in the impedance, at the natural frequencies of standing waves in the plasma.

A similar model of the plasma is used in this investigation, mainly to obtain a tractable mathematical problem. The plasma is again supposed to be homogeneous ana contained hetween two paralitel planes, the boundaries of the "plasma slab". It is the interaction with electromagnetic waves, propagating at an angle to the slab normal, which is of intcrcot hore, so this is not a one-dimensional problem in contrast to the "plasma capacitor". The electrons are again supposed to be confined to the slab by specular reflection at the slab boundaries. This can be thought of as due to a large static clcctric field very near the boundaries, similar to the boundary sheath field in a laboratory discharge plasma. The ions can be supposed motionless; they simply proviae a uniform neubrulizing backgroumd of charge on the time scale of interest (the plasma oscillation period, $2 \pi / \omega_{p}$ ). Because of the quasi-neutrality of the plasma, there is no static electric field in the plasma except very near the boundaries. The electrons, therefore, travel on trajectories which are very nearly straight lines until 
they are specularly reflected at the boundaries, being slightly perturbed by the electric fields of plasma waves and by particle encounters.

An essential point in the present calculation of the absorption and emission from the plasma slab is that a boundary-value problem has been solved for the electromagnetic fields. The usual method of calculating emission from a plasma makes use of the radiative transfer equation (8), which is hased upon the geometrical optics approximation. In the case of most interest in this investigation, the thickness of the plasma slab is less than the wavelength of the electromagnetic waves. Hence, the approximation of geometrical optics is invalid and the radiative transfer equation cannot be used. Also, the reflection of waves at the boundaries of a plasma cannot be correctly accounted for in the usual approach unless the waves lose phase information at each reflection. Furthermore, there is implicit in the radiative transfer approach the assumption of small damping. The solution of the boundary-value problem used here has none of these shortcomings.

In Part II the boundary-value problem for the plasma slab is solved. The self-consistent electric field in the plasma is obtained in terms of the boundary values of the tangential component of magnetic field. Maxwell's equations are Fourier analyzed in Section A and the particle equations of motion solved approximately in Section $B$. Then the Fourier coeffieients of the current density are obtained in Section $\mathrm{C}$, in terms of the electric field in the plasma, from the particle motions. In section $D$ the self-consistent field is solved for. 
This is the fiela which causes the particles to produce a current density which generates the same field, through the solution of Maxwell's equations. The steps in Sections $B$ and $C$ are usually carried out by means of the Vlasov equation in most theoreticai treatments of the self-consistent field in a hot plasma (9). The treatment given here brings out more clearly the physical content of the theory and expresses the assumptions and approximations involved in terms of the particle trajectories, rather than the distribution function. Tnis method is equivalent to the usual method, however, because the differential equations for the particle trajectories are the same as the Lagrangian subsidiary equations, or characteristics, of the Vlasov equation (10).

The solution of the boundary-value problem is used in Part III to find the absorption coefficient of the plasma slab, i.e., the fraction of the power in an incident electromagnetic wave which is absorbed. The wave is assumed to be polarized with $\vec{E}$ in the plane of incidence so that it excites longitudinal plasma waves in the slab. In Section A the concept of surface impedance is introduced which is a ratio of fields at the surface of the plasma and is obtained from the solution of the boundary-value problem. The absorption coefficient is related to it in section B and, therefore, to the properties of the plasma. The general expression for the absorption coefficient, equation 3.24, is interpreted in terms of Landau dampine of plasma waves. If the frequency of the incident wave is close to the natural frequency for a longitudinal plasma wave with an integral number of halfwavelengths between the siab boundaries, then such a standing wave can 
be excited in the plasma by the incident wave. Because the plasma wave is Landau-damped, the plasma absorbs energy from the incident wave. Under some conditions, discussea in Section $C$, the absorption is appreciable but only for frequencies very near these "normal mode" I'requencies. 'L'he absorption spectrum tinen contains narrow lines at these frequencies, with Lorentzian shapes. The reflectivity of the plasma slab, i.e. the fraction of power in the incident wave which is refilected, also has resonances at these frequencies. In section $D$ the notion of Landau damping in the plasma slab is discussed critically, as well as the conditions under which it can be the primary mechanism for power absorption in a steady state.

In Part IV the statisticai properties of the noise radiation from a plasma slab are calculatea. In Section A the surface emissivity is defined, and related to the autocorrelation function of the radiation fiela. It is then shown to be related to the Laplace transform of the radiation field which is more easily obtained than the timedependent field and which is related to the Laplace transform of the field fluctuations at the plasma boundary. These boundary values are determined in Section B by the solution of a boundary-value problem for the electric Iield Iluctuations in the plasma. The results of Part II are simply carried over, with a reinterpretation of some of the variables. The field fluctuations are produced by "dressed test particles" I.e., individual charged particles and the1r screening clouds, which are assumed to be statistically independent. The generai expression for the emissivity, equation 4.18 , is then derived in a straightforward way. The radiation from the slab is just a result of continuing the 
field fluctuations in the plasma into the region external to the plasma by requiring continuity of the fields at the boundary. The emission mechanism is therefore the excitation of electric field fluctuations by the motions of the indiviaual charged particles. Emission lines may occur, under some conditions, at the plasma wave resonance frequencies, the emissivity being proportional to the numider of particles with velocities close to the phase velocities of the waves. The enhancement of emission lines, caused by an increase in the number ot fast electrons over a thermal equilibrium distribution, is discussed in Section C. The effect of particle encounters, or collisions, is examined briefly in Section D.

Finaliy, the results are summarizeà in Part $V$ and compared with the experimental results on microwave scattering, absorption, and emission by gaseous discharge plasmas. 
II. SELF-CONSISTENT SOLUTION OF MAXWELI'S EQUATIONS AND

THE PARTICLE EQUATIONS OF MOTION

\section{A. Fourier Analysis of Maxweli's Equations}

As a first step in the self-consistent solution of the differential equations for the eiectromagnetic fields and the charged particles, we suppose the current density $\vec{j}(\vec{x}, t)$ to be krown; the electromagnetic fields are then determined by the solution of Maxwell's equations:

$$
\begin{aligned}
& \operatorname{curl} \vec{B}=\frac{4 \pi}{c} \vec{j}+\frac{1}{c} \frac{\partial \vec{E}}{\partial t} \\
& \operatorname{curl} \vec{E}=-\frac{1}{c} \frac{\partial \vec{B}}{\partial t}
\end{aligned}
$$

The soiution of these equations in the plasma volume $V$ for all times $t>0$ can be made unique (II) by specifying (in adaition to the current density in $V$ for $t \geq 0$ ) the tangential components of $\vec{B}$ on the surface $S$ of the plasma voiume, for $t \geqslant 0$, and the initial values (at $t=0$ ) of $\vec{E}$ and $\vec{B}$ at all points of $V$ whose distances from the observation point are $\leq \mathrm{ct}$.

Although we have to solve an initial boundary-value problem in principle, we are most interested in tine dependence of the solution upon the boundary values of tangential $\bar{B}$. This is because we are interested in steady-state, or time-stationary phenomena, for which the initial values have no effect. For simpiicity we will assume that $\operatorname{curl} \overrightarrow{\mathrm{E}}$ and $\overrightarrow{\mathrm{B}}$ are zero everywhere in $\mathrm{V}$ at $t=0$. The initial value of div $\overrightarrow{\mathrm{E}}$ is determined hy the charge density $\rho$ at $t=0$ by the relation aiv $\vec{E}=4 \pi \rho$, which we assume is satisfied at $t=0$. 
Laplace transforms of the field variables with respect to time may be introaced as follows:

$$
\vec{E}(\vec{x} ; \omega)=\int_{0}^{\infty} d t e^{i \omega t} \vec{E}(\vec{x}, t)
$$

and similarly for $\vec{B}$, where the usual Laplace transform variable $\mathbf{s}$ is replaced by $-i \omega$, and we take $\operatorname{Im} \omega>0$ to insure convergence of the integral. Laplace transforming Maxwell's equations yields the following equations for the transforms of the field variables:

$$
\begin{aligned}
& \operatorname{curl} \vec{B}=\frac{1}{c}(4 \pi \vec{j}-\vec{E}(0))-i k_{0} \vec{E} \\
& \operatorname{curl} \vec{E}=i k_{0} \vec{B} .
\end{aligned}
$$

where $x_{0}=\omega / c$, and $\vec{E}(0) \equiv \vec{E}(\vec{x}, t=0)$ is the initial condition on the electric rield.

Now the plasma volume $V$ will be chosen, for simplicity, to be a slab of thickness $L$, described by rectangular coordinates $x, y, z$ in the range $0 \leqslant x \leqslant I,-\infty<y<\infty,-\infty<z<\infty$. It is convenient to use Fourier integral representations of tine fiela variables with respect to $\mathrm{y}$ and $\mathrm{z}$, and Fourier series representations with respect to $x$ in the above interval. For the variables $E_{x}, B_{y}, B_{z}$, anc $j_{x}$ we use a sine-series represcntation, e.g.,

$$
E_{x}(x, y, z ; \omega)=\int_{-\infty}^{\infty} \frac{d k y}{2 T} \int_{-\infty}^{\infty} \frac{d k}{2 \pi} e^{i\left(k y+k_{z} z\right)} \frac{2}{L} \sum_{n=1}^{\infty} E_{x s}\left(\vec{k}_{n}, \omega\right) \sin k_{n} x
$$

where $\vec{k}_{n}=\left(k_{n}, k_{y}, k_{z}\right)$ and $k_{n}=n \pi / L$. For the variables $E_{y}, E_{z}$, 
$B_{x}, j_{y}, j_{z}$ and $\rho$, we use a cosine series representation, e.g.,

$$
E_{y}(x, y, z ; \omega)=\int_{-\infty}^{\infty} \frac{a k y}{2 \pi} \int_{-\infty}^{\infty} \frac{d k z}{2 \pi} e^{i\left(k y+x_{z} z\right)} \frac{2}{L} \sum_{n=0}^{\infty} E_{y c}\left(\vec{k}_{n}, \omega\right) \cos k_{n} x
$$

where the prime on the summation means the $n=0$ term is multiplied by $\frac{1}{2}$. The Fourier coefficients of the variables are obtained by integrating over the slab volume, e.g.

$$
\left\{\begin{array}{c}
E_{x s}\left(\vec{k}_{1 z}, \omega\right) \\
E_{y c}\left(\vec{k}_{n}, \omega\right)
\end{array}\right\}=\int_{-\infty}^{\infty} d y \int_{-\infty}^{\infty} d z e^{-i\left(k y y+k_{z} z\right)} \int_{0}^{L} d x\left\{\begin{array}{l}
E_{x}(\vec{x} ; \omega) \sin k_{n z} x \\
E_{y}(\vec{x} ; \omega) \cos k_{r_{z} x}
\end{array}\right\}
$$

Introduction of these Fourier representations into the $x, y$ and $z$ components of equation 2.4 yields the following equations:

$$
\begin{aligned}
k_{y} E_{z c}-k_{z} E_{y c} & =k_{o} B_{x c} \\
i k_{z} E_{x s}+k_{n} E_{z c} & =i k_{0} B_{y s} \\
-k_{n} E_{y c}-i k_{y} E_{x s} & =i k_{o} B_{z s}
\end{aligned}
$$

Similarly, from the $x$ component of equation 2.3 we obtain

$$
i k_{y} B_{z S}-i k_{z} B_{y s}=\frac{I}{c}\left(4 \pi j_{x S}-E_{x S}(0)\right)-i k_{0} E_{x S}
$$

If the $y$ and $z$ components of equation 2.3 are multipliea by $e^{-i\left(k y+y^{y}+\right)} \cos k_{n} x$ and integrated over the slab volume, the derivatives can be transformed by integration by parts. We obtain, in this way, the following equations: 


$$
\begin{aligned}
& i k_{z} B_{x c}-k_{n} B_{z s}+B_{z o}-(-1)^{n_{B} B_{z L}}=\frac{1}{c}\left(4 \pi j j_{y c}-E_{y c}(0)\right)-i k_{o} E_{y c} \\
& k_{n} B_{y s}-1 k_{y} B_{x c}-B_{y o}+(-1)^{n_{B}}{ }_{y L}=\frac{1}{c}\left(4 \pi j_{z c}-E_{z c}(0)\right)-i k_{0} E_{z c}
\end{aligned}
$$

where $\mathrm{B}_{\mathrm{zO}} \equiv \mathrm{B}_{\mathrm{z}}\left(0 ; \mathrm{k}_{\mathrm{y}}, \mathrm{k}_{\mathrm{z}}, \omega\right)$ and $\mathrm{B}_{\mathrm{zL}} \equiv \mathrm{B}_{\mathrm{z}}\left(\mathrm{L} ; \mathrm{k}_{\mathrm{y}}, \mathrm{k}_{\mathrm{z}}, \omega\right)$ are the Fourier transforms with respect to $y$ and $z$ of the boundary values of $B_{z}$ at $x=0$ and $x=I$. The occurrence of these boundary terms is a result of integration by parts in the term containing the derivative. $\frac{\partial \mathrm{B}_{z}}{\partial \mathrm{x}}$

The Fourier coefficients of $\vec{B}$ may be eliminated from the above six equations. The resulting three equations for the Fourier coefficients of $\vec{E}$ can be written as follows (where we consider only the $\mathrm{k}_{\mathrm{z}}=0$ componerts ):

$$
\begin{gathered}
\left(k_{0}^{2}-k_{y}^{2}\right) E_{x s}+i k_{n} k_{y} E_{y c}=-\frac{i k_{0}}{c}\left(4 \pi j_{x s}-E_{x s}(0)\right) \\
-i k_{n} k_{y} E_{x s}+\left(k_{0}^{2}-k_{n}^{2}\right) E_{y c}=-\frac{i k_{0}}{c}\left(4 \pi j_{y c}-E_{y c}(0)\right) \\
+i k_{0}\left[B_{z o}-(-1)^{n_{B}} B_{z L}\right] \\
{\left[i_{0}^{2}-\left(k_{n}^{2}+k_{y}^{2}\right)\right] E_{z c}=-\frac{i k_{0}}{c} 4 \pi j_{z c}-i k_{0}\left[B_{y o}-(-1)^{n_{B}}\right]}
\end{gathered}
$$

The last equation is uncoupled from the first two by the choice $\mathrm{k}_{\mathrm{z}}=0$, which means simply that the fieids do not vary in the $\mathrm{z}$ direction. Also, $E_{z c}(0)=0$ in that case.

If the fourier coetiticients of $\vec{j}$ were known, then the solution of these equations for $E_{x s}, E_{y c}$ and $E_{z c}$ would give the Fourier 
representation of the electric field inside the slab in terms of the bounaary values of tangential $\vec{B}$ at the surface. But the current density is determined by the motions of the charged particles of the plasma, which are influenced by the electric field in the plasma. Therefore, the next step in the self-consistent determination of the field is to find the current density $\vec{j}$ produced by a known field $\vec{E}$. B. Perturbation Analysis of the Particle Equations of Motion Before the current density can be founa, the motions of the charged particies in a known field $\overrightarrow{\mathrm{E}}$ must be determired. The position $\vec{r}(t)$ of a particle with (charge/mass) $=n$ in an electric fiela $\overrightarrow{\mathrm{E}}(\overrightarrow{\mathrm{x}}, t)$ is determined by the solution of the differential equation of motion:

$$
\ddot{\vec{r}}=\eta \overrightarrow{\mathrm{E}}(\vec{r}(t), t)
$$

The solution is made unique by specifying initial conditions:

$$
\vec{r}(0)=\vec{r}_{0}, \overrightarrow{\vec{r}}(0)=\vec{v}_{0}
$$

We assume the electric field to be given by

$$
\vec{E}(\vec{x}, t)=\vec{E}_{0}(\vec{x}, t)+\vec{E}_{l}(\vec{x}, t)
$$

so that

$$
\ddot{\vec{r}}(t)=n \overrightarrow{\mathrm{E}}_{0}(\vec{r}(t), t)+\eta \overrightarrow{\mathrm{E}}_{I}(\vec{r}(t), t)
$$

where $\eta \vec{E}_{1}$ is a smali perturbation in the equation of motion. Because this differential equation is nonlinear, we need to use approximation methods. The solution may be obtaincd as a porturbation series: 


$$
\vec{r}(t)=\vec{r}^{-13-}(0)(t)+\vec{r}^{(1)}(t)+\cdots
$$

where $\vec{r}^{(0)}(t)$ is the solution of the equation of motion with $n \vec{E}_{1}$ negiected:

$$
\ddot{\vec{r}}^{(0)}(t)=n \overrightarrow{\mathrm{E}}_{0}\left(\overrightarrow{\mathrm{r}}^{(0)}(t), t\right)
$$

For simplicity we require that $\vec{r}^{(0)}(t)$ have the same initiai conditions (equation 2.9) as $\vec{r}(t)$, so that the other terms in the perturbation series satisfy zero initial conditions, e.g.

$$
\vec{r}^{(1)}(0)=0, \quad \dot{\vec{r}}^{(1)}(0)=0
$$

The solution of the nonlinear equation 2.12 with initial conditions 2.9 is assumed to be a known function. For definiteness we denote this function as follows:

$$
\vec{r}^{(0)}(t)=\vec{R}\left(t \mid \vec{r}_{0}, \vec{v}_{0}, 0\right)
$$

where the initial conditions are indicated explicitly.

At this point we digress to discuss in some detail the trajcctory function $\overrightarrow{\mathrm{B}}$ for a particular case to which the analysis will be applied. We will aeal with a model of a nearly-collisionless hot plasma confined to a siab with boundary planee $x=0$ and $x-L$. The ions can bc supposed nearly motioniess (on the time scales of interest) and the electrons confined by an equilibrium static field $\overrightarrow{\mathrm{E}}_{\mathrm{O}}(\overrightarrow{\mathrm{x}})$ given by

$$
n \vec{E}_{0}(\vec{x})=-\hat{x} \frac{a \phi_{0}(x)}{d x} \text {, where } \emptyset_{0}(x)=\emptyset_{0} \cot ^{2}\left(\frac{\pi x}{L}\right)
$$

and $n=-e / m_{e}$ for electrons. The potential energy/unit mass $\emptyset_{0}(x)$ 
has the form of a well, with sides which become infinitely high as the boundaries are approached from inside the slab.

Since the ejectrons are not confined in the $y$ and $z$ directions, the trajectory function may be written

$$
\vec{R}\left(t \mid \vec{r}_{0}, \vec{v}_{0}, 0\right)=\left[X\left(t \mid x_{0}, v_{x 0}, 0\right), y_{0}+v_{y 0} t, z_{0}+v_{z o} t\right] .
$$

The function $X$ may be obtained from the energy integral,

$$
\mathcal{U}=\frac{v_{\mathrm{x}}^{2}}{2}+\phi_{0} \cot ^{2}\left(\frac{\pi \mathrm{x}}{\mathrm{L}}\right)=\frac{\mathrm{v}_{\mathrm{xO}}^{2}}{2}+\phi_{0} \cot ^{2}\left(\frac{\pi \mathrm{x}}{\mathrm{L}}\right) .
$$

It is given explicitly by

$$
\begin{aligned}
x\left(t \mid x_{0}, v x_{0}, 0\right)=\frac{L}{\pi} \cos ^{-1} & \left\{\cos \left(\frac{\pi x_{0}}{L}\right) \cos \Omega t .\right. \\
& \left.\mp \sqrt{\frac{1}{1+\phi_{0} / u}-\cos ^{2}\left(\frac{\pi x_{0}}{L}\right)} \sin \Omega t\right\}
\end{aligned}
$$

where $\Omega(\mathcal{U})=\sqrt{2} \frac{\pi}{L}\left(\mathcal{U}+\emptyset_{0}\right)^{I / 2}$ is the fundamental oscillatior frequency in the potential well, as a function of energy, and $\mathcal{U}$ is given in terms of $x_{0}$ and $v_{x o}$ by equation 2.16. The value of lise irserse cosine is chosen to lie always between 0 and $\pi$, and the upper (lower) sign in front of the square root is taken for $v_{x o}>0$ $\left(v_{x O}<0\right)$.

We will treat only the limitirg case $\phi_{0} \rightarrow 0$ for which the potential approaches a square well, and the oscillation frequency becomes $\Omega=\frac{\pi}{L}\left|v_{x o}\right|$. Then equation 2.17 becomes, in this limit, 


$$
x\left(t \mid x_{0}, v_{x 0}, 0\right)=\frac{I}{\pi} \cos ^{-1}\left\{\cos \frac{\pi}{L}\left(x_{0}+v_{x o} t\right)\right\} .
$$

Since the confining field acting on the particle is negligible except very near the boundaries, the particle moves on a straight-line trajectory with a constant speed until it reaches one of the boundary surfaces, where it is specularly reflected. The $\mathrm{x}$ component of velocity appears to change sign discontinuously in this limit, since differentiation of equation 2.18 gives

$$
\dot{x}\left(t \mid x_{0}, v_{x_{0}}, 0\right)=v_{x_{0}} \frac{\sin \frac{\pi}{L}\left(x_{0}+v_{x_{0}} t\right)}{\left|\sin \frac{\pi}{L}\left(x_{0}+v_{x_{0}} t\right)\right|}
$$

Whenever a continuous function is needed to justify the analysis below, we can always return, in principle, to equation 2.17 with $\emptyset_{0} / \mathscr{U}<1$.

Returning now to the discussion of the particle equation of motion including the small perturbation $\vec{n}_{\mathrm{E}_{1}}$, we substitute the perturbation series 2.11 into equation 2.10. After expanding $\vec{E}_{0}(\hat{r}(t), t)$ in a raylor series about $\vec{r}(t)=\vec{r}^{(0)}(t)$, we find that $\vec{r}^{(1)}(t)$ satisfies the following equation (in Cartesian tensor notation):

$$
\ddot{r}_{\mu}^{(I)}(t)-\eta \nabla_{\alpha} E_{O \mu}\left(\vec{r}^{(0)}(t), t\right) r_{\alpha}^{(I)}(t)=\eta E_{I \mu}\left(\vec{r}^{(0)}(t), t\right) .
$$

[The higher order terms in the perturbation series satisfy inear differential equations like 2.20, but with right-hand sides of correspondingly nigher order in $n E_{1}$; these will not be considered here.] 
The solution of equation 2.20 with zero initial conditions can be written in the form

$$
r_{\mu}^{(I)}(t)=n \int_{0}^{t} a t^{\prime} T_{\mu \beta}\left(t, t^{\prime}\right) E_{I B}\left(\vec{r}^{(0)}\left(t^{\prime}\right), t^{\prime}\right) .
$$

The tensor $T_{\mu \beta}$ is assumed to satisfy the differential equation

$$
\ddot{T}_{\mu \beta}\left(t, t^{\prime}\right)-\eta \nabla_{\alpha} E_{O \mu}\left(\vec{r}^{(0)}(t), t\right) T_{\alpha \beta}\left(t, t^{\prime}\right)=0
$$

(where dots indicate differentiation with respect to $t$ ) and the initial conāitions

$$
T_{\mu \beta}=0, \quad \dot{T}_{\mu \beta}=\delta_{\mu \beta} \quad \text { at } t=t^{\prime} \text {. }
$$

Now we recall that the trajectory function $\vec{R}\left(t \mid \vec{r}^{\prime}, \vec{v}^{\prime}, t^{\prime}\right)$ satisfies the differential equation

$$
\ddot{R}_{\mu}-\eta E_{O ; \mu}(\vec{R}, t)=0
$$

and the initial conditions $R_{\mu}=r_{\mu}^{\prime}, \dot{R}_{\mu}=v_{\mu}^{\prime}$ at $t=t^{\prime}$. Differentiating with respect to $v_{\beta}^{\prime}$ gives the differential equation 2.22 anc the initial conditions 2.23. Hence $\Gamma_{\mu \beta}$ is given expiicitly in terms of the known trajectory function $\vec{R}$ by

$$
\mathrm{T}_{\mu \beta}\left(t, t^{\prime}\right)=\frac{\partial}{\partial v_{\beta}^{\prime}} R_{\mu}\left(t \mid \vec{r}^{\prime}, \vec{v}^{\prime}, t^{\prime}\right) .
$$

We have assumed, of course, that $\vec{r}^{\prime}=\vec{r}^{(0)}\left(t^{\prime}\right), \vec{v}^{\prime}=\dot{\vec{r}}^{(0)}\left(t^{\prime}\right)$, so that $\vec{R}\left(t \mid \vec{r}^{\prime}, \vec{v}^{\prime}, t^{\prime}\right)=\vec{r}^{(0)}(t)$. 
Thus the solulion of the linearized equation of motion 2.20 for the deviation of the particle trajectory from the unperturided trajectory $\vec{r}^{(0)}(t)$ is given by equations 2.21 and 2.25 as an integral of the perturbation $n \vec{E}_{1}$ experienced by a particle which moves along the unperturbed trajectory from time 0 to $t$. How much the trajectory is deviatea oy a given value of $n \overrightarrow{\mathrm{E}}_{l}$ is determined by tine influence tensor $T_{\mu \beta}$, which is determined by the unperturbed trajectory. For tine example considered above, we have

$$
\begin{aligned}
T_{x \beta}\left(t, t^{\prime}\right) & =\delta_{x \beta} \frac{\partial}{\partial v_{x}^{\prime}} x\left(t \mid x^{\prime}, v_{x}^{\prime}, t^{\prime}\right) \\
& =\delta_{x \beta}\left(t-t^{\prime}\right) \frac{\sin \frac{\pi}{L}\left(x^{\prime}+v_{x}^{\prime}\left(t-t^{\prime}\right)\right)}{\left|\sin \frac{\pi}{L}\left(x^{\prime}+v_{x}^{\prime}\left(t-t^{\prime}\right)\right)\right|} .
\end{aligned}
$$

If the particle undergoes an even number of reflections at the boundaries between $t$ imes $t^{\prime}$ and $t$, then a positive perturbation $n E_{I x}\left(\vec{r}^{\prime}, t^{\prime}\right)$ causes a positive contribution to $x^{(I)}(t)$ but if the number of reflections is oad, the contribution is negative.

Finally, a few remarks are necessary concerning the linearization of the particie equations of motion. The electric field perturbation $\overrightarrow{\vec{\Xi}}_{1}$ wilz be interpreted as a wave with frequency close to the plasma frequency $\omega_{p}$. In order that collective behavior with this ciaracteristic frequency be correctly aescribed by the linearized equations, the wave amplitude must be small enough that the particles are not deviated appreciably from their unperturbed trajectories during a time interval of many plasma oscillation periods. Furthermore, close-range encounWers velween particies must lue assumed to occur infrequently enough, 
and the cumulative effect of long-range encounters assumed slow enough that the unperturbed trajectories provide a good description of the motion of a particle for many piasma periods. A rough criterion is that $\omega_{p} t_{c} \gg I$, where $t_{c}$ is the collisional relaxation time (see Section IV,D.) On the other hand, the collision rate must be sufficiently high to prevent the invalidation of the linearized equations due to particle trapping. This will be discussed further in Section III,D.

\section{Fourier Coefficients of the Current Density}

The current density due to the electrons is

$$
\vec{j}(\vec{x}, t)=(-e)\left\langle\sum_{j} \vec{v}_{j}(t) \delta\left(\vec{x}-\vec{r}_{j}(t)\right)\right\rangle
$$

where $\vec{r}_{j}(t)$ and $\vec{v}_{j}(t)$ are the position and velocity of the $j^{\text {th }}$ particle. The ensemble average, indicated by the angular brackets, is taken because we are interested here in the macroscopic current produced by a known fieid $\vec{E}$, and not in the fluctuations due to particle discreteness. Hence, the positions and velocities of the particles at time $t$ are treated as random variables with a known joint probability distribution, and the brackets indicate that we use the statistical mean over all possible positions and veiocities of all particles. 


$$
\vec{j}\left(\vec{k}_{n}, t\right)=\int_{-\infty}^{\infty} d y \int_{-\infty}^{\infty} d z e^{-i\left(k_{y} y+k_{z} z\right)} \int_{0}^{L} d x\left\{\begin{array}{l}
j_{x}(\vec{x}, t) \sin k_{n} x \\
j_{y}(\vec{x}, t) \cos k_{n} x \\
j_{z}(\vec{x}, t) \cos k_{n} x
\end{array}\right\}
$$

Using equation 2.27 thio may be written

$$
j_{\alpha}\left(\vec{k}_{n}, t\right)=(-e)\left\langle\sum_{j} v_{j \mu}(t) g_{\mu \alpha}\left(\vec{r}_{j}(t), \vec{k}_{n}\right)\right\rangle
$$

where $g_{\mu \alpha}=0$ for $\alpha \neq \mu$ and

$$
\left.\begin{array}{l}
g_{x x}\left(\vec{x}, \vec{k}_{n}\right)=e^{-i k y} \sin k_{n} x \\
g_{z z}\left(\vec{x}, \vec{k}_{n}\right)=e^{-i k_{y} y} \cos k_{n} x
\end{array}\right\} \text { for } k_{z}=0
$$

Now the perturbation series of the previous section can be used for $\vec{r}_{j}(t)$ and $\vec{v}_{j}(t) \equiv \vec{r}_{j}(t)$, and if we retain terms up to first order in $\eta \Psi_{1}$, we have approximately

$$
\begin{aligned}
\dot{u}_{\alpha}\left(\vec{k}_{n}, t\right)=(-e)\left\langle\sum_{j}\right. & \left\{v_{j \mu}^{(0)}(t) g_{\mu \alpha}\left(\vec{r}_{j}^{(0)}(t), \vec{k}_{n}\right)+v_{j \mu}^{(I)}(t) g_{\mu \alpha}\left(\vec{r}_{j}^{(0)}(t), \vec{k}_{n}\right)\right. \\
& \left.\left.+v_{j \mu}^{(0)}(t) r_{j Y}^{(I)}(t) \nabla_{\gamma} g_{\mu \alpha}\left(\vec{r}_{j}^{(0)}(t), \vec{k}_{n}\right)\right\}\right\rangle
\end{aligned}
$$

The first term on the right-hand side of this equation is a current density Fourier component for particles unperturbed by the fieid $n \mathrm{E}_{I}$ and may be denoted by $\vec{j}^{(0)}\left(\vec{k}_{n}, t\right)$ :

$$
j_{\alpha}^{(0)}\left(\vec{k}_{n}, t\right)=(-e)\left\langle\sum_{j} v_{j \mu}^{(0)}(t) g_{\mu \alpha}\left(\vec{r}_{j}^{(0)}(t), \vec{k}_{n}\right)\right\rangle
$$


The second and third terms contain the perturbation in the position and velocity due to the field $\overrightarrow{\mathrm{E}}_{1}$. From equations 2.21 and 2.25 these perturbation quantities are

$$
r_{\mu}^{(I)}(t)=n \int_{0}^{t} d t^{\prime} E_{1 B}\left(\vec{r}^{\prime}, t^{\prime}\right) \frac{\partial}{\partial v_{\beta}^{\prime}} \cdot R_{\mu}\left(t \mid \vec{r}^{\prime}, \vec{v}^{\prime}, t^{\prime}\right)
$$

and

$$
v_{\mu}^{(I)}(t)=n \int_{0}^{t} d t^{\prime} E_{I \beta}\left(\vec{r}^{\prime}, t^{\prime}\right) \frac{\partial}{\partial v_{\beta}^{\prime}} \dot{R}_{\mu}\left(t \mid \vec{r}^{\prime}, \vec{v}^{\prime}, t^{\prime}\right)
$$

where $\vec{r}^{\prime}=\vec{R}\left(t^{\prime} \mid \vec{r}_{0}, \vec{v}_{0}, 0\right) \quad, \vec{v}^{\prime}=\dot{\vec{R}}\left(t^{\prime} \mid \vec{r}_{0}, \vec{v}_{0}, 0\right)$

are the unperturbed position anō velocity at time $t^{\prime}$. (The particle index $j$ has been dropped for simplicity). The second term in equation 2.28 may be written as follows:

$$
\begin{aligned}
v_{\mu}^{(I)}(t) g_{\mu \alpha}\left(\vec{r}^{(0)}(t), \vec{k}_{11}\right) & =n \int_{0}^{t} d t^{\prime} E_{1 \beta}\left(\vec{r}^{\prime}, t^{\prime}\right) \\
& \frac{\partial}{\partial v_{B}^{\prime}} \dot{R}_{\mu}\left(t \mid \vec{r}^{\prime}, \vec{v}^{\prime}, t^{\prime}\right) g_{\mu \alpha}\left(\vec{R}\left(t \mid \vec{r}^{\prime}, \vec{v}^{\prime}, t^{\prime}\right), \vec{k}_{n}\right)
\end{aligned}
$$

The argument of the functions $g_{u \alpha}$ is $\vec{r}(0)(t)=\vec{R}\left(t \mid \vec{r}^{\prime}, \vec{v}^{\prime}, t^{\prime}\right)$ where $t^{\prime}$ is the integration variable. Note that the value of $\vec{R}\left(t \mid \vec{r}^{\prime}, \vec{v}^{\prime}, t^{\prime}\right)$ is constant (for $t$ fixed) and dues nul change with $b^{\prime}$ as long as $\vec{r}^{\prime}$ and $\vec{v}^{\prime}$ are given by equation 2.29 . The value of the integral is therefore not altered by formaliy including the functions $g_{\mu \alpha}$, with the above argument under the integration. The third term in equation 2.28 may be treated similarly, and combined with the second term by identifying the derivative of a product. The result is 


$$
\begin{aligned}
& v_{\mu}^{(L)}(t) E_{\mu \alpha}\left(\vec{r}^{(0)}(t), \vec{k}_{n}\right)+v_{\mu}^{(U)}(t) r_{\gamma}^{(L)}(t) \nabla_{\gamma} g_{\mu \alpha}\left(\vec{r}^{(U)}(t), \vec{k}_{n}\right) \\
& \quad t \\
& =n \int_{0} d t^{\prime} E_{I \beta}\left(\vec{r}^{\prime}, t^{\prime}\right) \frac{\partial}{\partial v_{\beta}^{\prime}}\left[\dot{R}_{\mu}\left(t \mid \vec{r}^{\prime}, \vec{v}^{\prime}, t^{\prime}\right) g_{\mu \alpha}\left(\vec{R}\left(t \mid \vec{r}^{\prime}, \vec{v}^{\prime}, t^{\prime}\right), \vec{k}_{r}\right)\right]
\end{aligned}
$$

When equation 2.31 is used in equation 2.28 , we sum over all particlcs and avcrage ovcr positions and velooities at time t'. We can use the probability density of unperturbed positions and velocities, $f_{0}\left(\vec{r}^{\prime}, \vec{v}^{\prime}, t^{\prime}\right)$, to first orajer in $\eta E_{I}$. To make the probability density properly normalized we must also divide by $V$, the voiume of Dlasma unaer consideraticn; the sum runs over the N particles in this volume. Since the particles are ali identical, the ensemble average of the sum is just $\mathbb{N}$ times the ensemile average for any one particle. Passing to the limit $N \rightarrow \infty, V \rightarrow \infty, N / V=n_{0}$ (finite), we obtair. the following from equation 2.28:

$$
\begin{aligned}
& j_{\alpha}\left(\vec{k}_{n}, t\right)=j_{\alpha}^{(0)}\left(\vec{k}_{n}, t\right)+\frac{n_{0} e^{2}}{m} \int_{0}^{l} d t^{\prime} \int d \vec{r}^{\prime} \int d \vec{v}^{\prime} f_{0}\left(\vec{r}^{\prime}, \vec{v}^{\prime}, t^{\prime}\right) \\
& \text { where }\left[E_{1 \beta^{\prime}}\left(\vec{r}^{\prime}, t^{\prime}\right) \frac{\partial}{\partial v_{\beta}^{\prime}}[,\right. \\
& =\left[\dot{k}_{\mu}\left(t \mid \vec{r}^{\prime}, \vec{v}^{\prime}, t^{\prime}\right) g_{\mu \alpha}\left(\vec{R}\left(t \mid \vec{r}^{\prime}, \vec{v}^{\prime}, t^{\prime}\right), \vec{k}_{n}\right)\right] .
\end{aligned}
$$

I'ne same result can be obtained from the linearized Vlasov equation(9) by integrating along unperturbed trajectories, integrating over velocity $\vec{v}$ to get $\vec{j}$, integrating over position $\vec{r}$ to get the Fourier coefficients, making a change of integration variables from $\vec{r}, \vec{v}$ to $\vec{r}^{\prime}, \vec{v}^{\prime}$ and integrating by parts with respect to $\vec{v}^{\prime}$. The 
method used above is somewhat more direct, however, and brings out the physical meaning of the linearization process, in terms of particle trajectories, more cleariy.

To proceed further it is necessary to make some further assumptions about the equilibrium state of the plasma. The model which will be used here is that of a homogeneous slab, with a time-independent equilibrium state. 'Then the particle position $\vec{r}$ ' is distributed (statistically) uniformly within the slab volume, and its probability density is independent of time:

$$
f_{0}\left(\vec{r}^{\prime}, \vec{v}^{\prime}, t^{\prime}\right)=\left\{\begin{array}{l}
f_{0}\left(\vec{v}^{\prime}\right), 0 \leq x^{\prime} \leq L \\
0, \text { otherwise }
\end{array}\right\}
$$

The trajectory function depends only on the difference between the initial and firal times: $\vec{R}\left(t \mid \vec{r}^{\prime}, \vec{v}^{\prime}, t^{\prime}\right)=\vec{R}\left(t-t^{\prime} \mid \vec{r}^{\prime}, \vec{v}^{\prime}, 0\right)$ and is given explicitly by equations 2.15 and 2.18 for the square well confining potential. Using these equations and equation 2.19 the components of the square bracket in equation 2.32 (except for the factor $\left.e^{-i k y\left(y^{\prime}+v_{y}^{\prime} \tau\right)}\right)$ can be written as foliows:

$\underline{\alpha=x \text { component }} \dot{X}\left(\tau \mid X^{\prime}, v_{X}^{\prime}, 0\right) \sin k_{n} X\left(\tau \mid X^{\prime}, v_{X}^{\prime}, 0\right)=v_{X}^{\prime} \sin k_{L i}\left(x^{\prime}+v_{X}^{\prime} \tau\right)$

$$
=v_{x}^{\prime}\left\{\sin k_{n} x^{\prime} \cos k_{n} v_{x}^{\prime} \tau+\cos k_{n} x^{\prime} \sin k_{n} v_{x}^{\prime} \tau\right\} .
$$

where $\tau=t-t^{\prime}$, and

$\underline{\alpha=y \text { component }} v_{\dot{y}}^{\prime} \cos k_{n} X\left(\tau \mid x^{\prime}, v_{x}^{\prime}, 0\right)=v_{y}^{\prime} \cos k_{n}\left(x^{\prime}+v_{x}^{\prime} \tau\right)$

$$
=v_{y}^{\prime}\left\{\cos k_{n} x^{\prime} \cos k_{n} v_{x}^{\prime} \tau-\sin k_{n} x^{\prime} \sin k_{n} v_{x}^{\prime} \tau\right\}
$$

(and similarly for the $\alpha=z$ component, with $v_{y}^{\prime}$ replaced by.$v_{z}^{\prime}$. ) 
Now the square bracket in equation 2.32 is differentiated with respect to the $B$ component of $\vec{v}^{\prime}$, multipliea by $f_{0}\left(\vec{v}^{\prime}\right)$, and integrated over all. $\vec{v}^{\prime}$. Assuming $f_{0}$ to be a function of the magnitude of $\vec{v}^{\prime}$ only (i.e., isotropic in velocity space), the terms which are odd functions of $v_{x}, v_{y}$ or $v_{z}$ will drop out upon integration. The surviving terms are proportional to either $\sin k_{n} x^{\prime}$ or $\cos k_{n} x^{\prime}$, ard so the integration over $\vec{x}^{\prime}$ in equation 2.32 transforms the components of $\overrightarrow{\mathrm{E}}_{1}$ into its Fourier coefficients. We obtain, finally

$$
\dot{j}_{\alpha}\left(\vec{k}_{n}, t\right)=j_{\alpha}^{(0)}\left(\vec{k}_{n, t}\right)+\int_{0}^{t} d t^{\prime} \sigma_{\alpha \beta}\left(\vec{k}_{n} ; t-t^{\prime}\right) E_{1 \beta}\left(\vec{k}_{n}, t^{\prime}\right)
$$

where

$$
\left[\sigma_{\alpha \beta}\left(\vec{k}_{n} ; \tau\right)\right]=\frac{\omega_{n}^{2}}{4 \pi} \int d \overrightarrow{v f}_{0}^{\prime}(\vec{v}) e^{-i\left(k_{n} v_{x}+k_{y} v_{y}\right) \tau}\left[\begin{array}{ccc}
\left(1-i k_{n} v_{x} \tau\right) & k v_{y} \tau & 0 \\
-k_{n} v_{y} \tau & \left(1-i k v_{y} \tau\right) & 0 \\
0 & 0 & 1
\end{array}\right]
$$

The fact that the Fourier coefficients of the current density are linearly related to those of the eiectric field, with the same wave vector $\vec{k}_{n}$, is a result of the choicc of a. homogencous piasma moacl, with simple specuiarly reflecting trajectories.

The Laplace transform of equation 2.33 can be obtained by using the convolution theorem; using $E_{\beta}\left(\vec{k}_{n}, w\right)$ fur line Laplace lransforil of $E_{I R}\left(\overrightarrow{\mathrm{x}}_{n}, t\right)$, we have

$$
\dot{j}_{\alpha}\left(\vec{k}_{n}, \omega\right)=j_{\alpha}^{(0)}\left(\vec{k}_{n}, \omega\right)+\sigma_{\alpha \beta}\left(\vec{k}_{n}, \omega\right) E_{\beta}\left(\vec{k}_{n}, \omega\right)
$$

where 


$$
\left[\sigma_{\alpha \beta}\left(\vec{k}_{n}, \omega\right)\right]=\frac{i \omega_{p}^{2}}{4 \pi} \int \overrightarrow{d v} \frac{f_{0}(\vec{v})}{\left(\omega-k_{n} v_{x}-x_{y} v_{y}\right)^{2}}\left[\begin{array}{ccc}
\omega-k_{y} v_{y} & i k_{y} v_{x} & 0 \\
-i k_{n} v_{y} & \omega-k_{n} v_{x} & 0 \\
0 & 0 & \omega-k_{n} v_{x}-k_{y} v_{y}
\end{array}\right]
$$

for $\operatorname{Im} \omega>0$.

This is the frequency and wave vector dependent conductivity tensor of the homogeneous plasma slab.

$$
\text { Using the assumed isotropy of } f(\vec{v}) \text {, the following relations are }
$$
easily derivea from equation 2.35:

$$
\begin{gathered}
\sigma_{x x}+\sigma_{y y}=\sigma_{\ell}+\sigma_{t}, \sigma_{x y}=-\sigma_{y x}, k_{n} \sigma_{x x}-i k_{y} \sigma_{x y}=k_{n} \sigma_{\ell}, \\
i k_{y} \sigma_{y y}+k_{n} \sigma_{x y}=i k_{y} \sigma_{\ell}
\end{gathered}
$$

where

$$
\sigma_{\ell}\left(\vec{k}_{n}, \omega\right)=\frac{i \omega \omega_{p}^{2}}{4 \pi} \int d \vec{u} \frac{f_{0}(\vec{u})}{\left(\omega-\vec{k}_{n} \cdot \vec{u}\right)^{2}}
$$

and

$$
\sigma_{t}\left(\vec{k}_{n}, \omega\right)=\sigma_{z z}\left(\vec{k}_{n}, \omega\right)=\frac{i \omega_{p}^{2}}{4 \pi} \int d \vec{u} \frac{f_{0}(\vec{u})}{\left(\omega-\vec{k}_{n} \cdot \vec{u}\right)}
$$

are the "Iongitudinal" and "transverse" conductivities. In the limit $($ Im $\omega) \rightarrow 0$, botì $\sigma_{t}$ anä $\sigma_{\ell}$ have real parts, which are obtaineà by using tre Dirac relation $1 / x+i \varepsilon=P \frac{1}{x}-i \pi \delta(x)$ for $\varepsilon \rightarrow 0$.

The above relations can be transformed into the following: 


$$
\begin{aligned}
& \sigma_{x x}=\alpha^{2} \sigma_{\ell}+\beta^{2} \sigma_{t}, \quad \sigma_{y y}=\beta^{2} \sigma_{\ell}+\alpha^{2} \sigma_{t} \\
& \sigma_{x y}=-\sigma_{y x}=i \alpha \beta\left(\sigma_{\ell}-\sigma_{t}\right),
\end{aligned}
$$

where

$$
\alpha=\frac{k_{n}}{\sqrt{k_{n}^{2}+k_{y}^{2}}} \quad, \quad \beta=\frac{k_{y}}{\sqrt{k_{n}^{2}+k_{y}^{2}}}
$$

These are equivalent to the statement that the matrix $\left(\begin{array}{cc}\sigma_{x x} & \sigma_{x y} \\ \sigma_{y x} & \sigma_{y y}\end{array}\right)$ can be diagonalized by a unitary transformation defined by

the equations

$$
\left\{\begin{array}{c}
E_{\ell}=\alpha E_{x s}+i \beta E_{y c} \\
E_{t}=i \beta E_{x s}+\alpha E_{y c}
\end{array}\right\}
$$

its eigenvalues being $\sigma_{\ell}$ and $\sigma_{t}$. Note that these eigenvalues are not purely imaginary; the matrix is not anti-hermitian, as it wouid be for a dissipationless medium.

It is convenient to make the following approximation:

$$
\sigma_{t}\left(\vec{k}_{n}, \omega\right) \simeq \frac{i \omega_{p}^{2}}{4 \pi \omega}
$$

Thic approximation can bo juctified in the following way. The denominator in the integrand of equation 2.37 can be expandea, for smai $\vec{k}_{n} \cdot \vec{u} / u$. Since $f_{0}(\vec{u})$ is isotropic, $\langle\vec{u}\rangle=0$, and the terms neglected are of order $\left|\vec{k}_{n}\right|^{2}\left\langle v^{2}\right\rangle / w^{2}$. For larse $n$ such that $\left|\overrightarrow{\mathrm{k}}_{\mathrm{n}}\right|^{2} \sim \omega^{2} /\left\langle\mathrm{v}^{2}\right\rangle$, the approximation 2.40 is not valid, but then $\left|\vec{k}_{n}\right|^{2} / k_{0}^{2} i c^{2} /\left\langle v^{2}\right\rangle>1$, and $\left|1+\frac{4 \pi i}{\omega} \sigma_{t}\right| \lesssim 1$ for $\omega^{2} \gtrsim \omega_{p}^{2}$. Hence 
the expression $\left[\left|\vec{k}_{n}\right|^{2} / k_{0}^{2}-\left(1+\frac{4 \pi i}{w} \sigma_{t}\right)\right]^{-1}$ in which $\sigma_{t}$ will always occur, can be well approximated by using 2.40 for all $n$.

Aithough we will be interested in frequencies fairly close to the plasma frequency, it is interesting to note that for very low frequencies, $\omega^{2} \sim \frac{\left\langle v^{2}\right\rangle}{c^{2}} \omega_{p}^{2}$, we have $\left|7+\frac{4 \pi i}{\omega} \sigma_{t}\right| \sim \frac{\omega_{0}^{2}}{\omega^{2}} \sim\left|\vec{k}_{n}\right|^{2} / k_{0}^{2}$ for $\left|\vec{k}_{n}\right|^{2} \sim \omega^{2} /\left\langle v^{2}\right\rangle$, and so the approximation 2.40 cannot be used for all n . For frequencies this low, particles can pass through the layer of widtn $c / \omega_{p}$ near the boundaries, where the field is not zero, in one perind of the field variation or less. The result is that absorption of energy can occur in collisionless plasmas which corresponds to the anomalous skin effect in metals (12).

\section{Self-Consistent Solution of the Boundary Value Problem}

The requirement that the electric field in the plasma be determined self-consistently means that the field--determined by equations $2.5,2.6$ and 2.7 in terms of the current density, the initial conditions, and tine boundary conditions-must be the same field which proulues the current densiby by equalion 2.34. Eliminating the Fourier components of the current density from these equations, we obtain the following:

$$
\begin{aligned}
\left(k_{0}^{2}-k_{y}^{2}+\frac{4 \pi i k_{0}}{c} \sigma_{x x}\right) E_{x s} & +\left(i k_{n} k_{y}+\frac{4 \pi i k_{0}}{c} \sigma_{x y}\right) E_{y c}= \\
& -i k_{0}\left(\frac{4 \pi}{c} j_{x s}^{(0)}-\frac{1}{c} E_{x s}(0)\right)
\end{aligned}
$$




$$
\begin{aligned}
& -2 T- \\
& \left(-i k_{n} k_{y}+\frac{4 \pi i k_{0}}{c} \sigma_{y x}\right) E_{x s}+\left(k_{0}^{2}-k_{n}^{2}+\frac{4 \pi i k_{0}}{c} \sigma_{y y}\right) E_{y c} \\
& =-i k_{0}\left(\frac{4 \pi}{c} j_{y c}^{(0)}-\frac{i}{c} E_{y c}(0)-B_{z o}+(-I)^{n} B_{z L}\right) \\
& \left(k_{0}^{2}-k_{n}^{2}-k_{y}^{2}+\frac{4 \pi i k_{0}}{c} \sigma_{z z}\right) F_{z c}=-i k_{0}\left(\frac{4 \pi}{c} j_{z c}(0)+R_{y o}-(-1)^{n_{B_{y}}}\right)
\end{aligned}
$$

The last equation can be solved immediately for $\mathrm{E}_{\mathrm{zc}}$. Using the approximation 2.40 for $\sigma_{z z}\left(=\sigma_{t}\right)$ we have

$$
E_{z c}=\frac{i k_{o}}{k_{n}^{2}-k_{p}^{2}}\left[\frac{4 \pi}{c} j_{z c}^{(0)}+B_{y o}-(-1)^{n} B_{y L}\right]
$$

where $\quad x_{p}^{2}=k_{0}^{2}\left(1-\omega_{p}^{2} / \omega^{2}\right)-k_{y}^{2}$

We recall that $j_{\mathrm{zc}}^{(0)}$ is the Fourier component of the $z$ component of the macroscopic current density which would exist in the absence of the field disturbance in the plasma, due to particles moving on their unperturbed trajectories. We will assume that this is zero ir problems involving excitation of macroscopic waves by boundary fields.

We now multiply by $(2 / L) \cos k_{n} x$ and sum over all $n$, leaving $k_{y}$ fixed, to get

$$
\begin{aligned}
E_{z}\left(x ; k_{y}, \omega\right)=\frac{2}{L} & \sum_{n=0}^{\infty} E_{z c}\left(\vec{k}_{n}, \omega\right) \cos k_{n} x= \\
& \frac{2 i k_{0}}{L} \sum_{n=0}^{\infty} \frac{\left(B_{y o}-(-1)^{n_{B}} B_{y L}\right) \cos k_{n} x}{k_{n}^{2}-k_{p}^{2}} .
\end{aligned}
$$


This is the Fourier transform with respect to $y$ and $z$ (with wave numbers $k_{y}$ and $k_{z}=0$ ) of the Laplace transform of the fiela $E_{z}$ at $x$. This Fourier series can be summed by complex variable techniques (13) with the resull

$$
E_{z}\left(x ; k_{y}, \omega\right)=\frac{-i k_{0}}{k_{p} \sin k_{p} L}\left[B_{y o} \cos k_{p}(x-L)-B_{y L} \cos k_{p} x\right]
$$

This result could have been obtained without the use of Fourier series methods by assuming that an electric field which is polarized perpendicular to the plane of incidence (the xy plane) propagates as though it were in a dielectric medium with index of refraction $\sqrt{1-\omega_{p}^{2} / \omega^{2}}$. This result is based on the approximation 2.40 and is therefore not valid for very low frequencies:

Equation 2.46 cannot be used when $k_{p} L$ is close to $\pi$ times an integer, because of the $\sin k_{p} L$ in the denominator. These combinations of $\omega$ and $k_{y}$ correspond to electromagnetic standing wave modes in the slab, such that a ronzero field can exist in the slab with zero boundary values. The response to given nonzero boundary values then becomes very large, in generai, as $k_{p} I \rightarrow m \pi$ (unless $B_{y o}=(-I)^{m_{H}}{ }_{y L}$ ). It is limited only by dissipative effects which have been neglectea in the approximations leading to equation 2.46 .

The z-component of the electric field will not de considered further, because it does not couple to the longitudinal plasma waves which are of primary interest.

We now return to the task of solving equations 2.41 and 2.42 . Using the reiations 2.38 and the approximation 2.40 , the determinant 
of the system of two equations can be shown to be

$$
a=k_{0}^{2}\left(I+\frac{4 \pi i}{\omega} \sigma_{\ell}\right)\left(k_{p}^{2}-k_{n}^{2}\right)
$$

where $k_{p}^{2}$ is given by equation 2.45. It is again necessary to require that $k_{p}^{2} \neq k_{n}^{2}$ so that the determinant is not zero; the solution is then straightforward. Defiring the longitudinal dielectric function $\varepsilon=1+\frac{4 \pi i}{\omega} \sigma_{\ell}$, we obtain the following:

$$
\begin{aligned}
E_{x s} / i k_{0}= & \frac{(4 \pi / c) i \beta j_{t}^{(0)}}{k_{n}^{2}-k_{p}^{2}}+\frac{4 \pi k_{n} \rho_{c}^{(0)}}{i k_{0}\left(k_{n}^{2}+k_{y}^{2}\right) \varepsilon\left(\vec{k}_{n}, \omega\right)} \\
& +i \alpha \beta\left(B_{z 0}-(-1)^{n_{B}}\right)\left[\frac{1}{k_{0}^{2} \varepsilon\left(\vec{k}_{n}, \omega\right)}+\frac{1}{k_{n}^{2}-k_{p}^{2}}\right] \\
E_{y c} / i k_{0} & =\frac{-(4 \pi / c) \alpha j_{t}^{(0)}}{k_{n}^{2}-k_{p}^{2}}-\frac{4 \pi k_{y} \rho_{c}^{(0)}}{k_{0}\left(k_{n}^{2}+k_{y}^{2}\right) \varepsilon\left(\bar{k}_{n}, \omega\right)} \\
& +\left(B_{z 0}-(-I)^{\left.n_{B} B_{z L}\right)}\left[\frac{\beta^{2}}{k_{0}^{2} \varepsilon\left(\vec{k}_{n}, \omega\right)}-\frac{\alpha^{2}}{k_{n}^{2}-k_{p}^{2}}\right]\right.
\end{aligned}
$$

where $j_{t}^{(0)}=i \beta j_{x s}^{(0)}+\alpha j_{y c}^{(0)}, \alpha$ and $\beta$ are defined in equation 2.38 and $\rho_{c}^{(0)}$ is the Fourier coefficient of a charge density for particles unperturbed by the electric field disturbance. We have used the continuity equation for this charge density

$$
\overline{a i v} \vec{j}^{(0)}+\frac{\partial \rho(0)}{\partial t}=0 \text {, or } k_{n} j_{x s}^{(0)}+i k_{y} j_{y c}^{(0)}-i \omega \rho(0)-\rho_{c}(0)=0 \text {, }
$$

the initial condition div $\vec{E}-4 \pi \rho=0$ at $t=0$, or 
$k_{n} E_{x s}(0)+i k_{y} E_{y c}(0)-4 \pi p_{c}(0)=0$ and the initial condition

$\operatorname{curl} \overrightarrow{\mathrm{E}}=0$ at $t=0$, or $k_{n} E_{\mathrm{yc}}(0)+i k_{\mathrm{y}} E_{\mathrm{xs}}(0)=0$.

We now assume that $j_{t}^{(0)}$ and $\rho_{c}^{(0)}$ are zero. The Fourier

series for a fixed value of $k_{y}$ are then

$$
\begin{aligned}
& E_{x}\left(x ; k_{y}, \omega\right)=-\frac{2 k_{y}}{k_{o}^{L}} \sum_{n=1}^{\infty} \frac{k_{n}\left(B_{z o}-(-I)^{n_{1}} B_{z I}\right) \sin k_{n} x}{\left(k_{n}^{2}+k_{y}^{2}\right) \varepsilon\left(\vec{k}_{n}, \omega\right)} \\
& -\frac{2 k^{k}{ }_{0}}{I} \sum_{n=1}^{\infty} \frac{k_{n}\left(B_{z 0^{-}}(-I)^{n_{B_{L}}}\right) \sin k_{n} x}{\left(k_{n}^{2}+k_{y}^{2}\right)\left(k_{n}^{2}-k_{p}^{2}\right)} \\
& F_{y}\left(x ; k_{y}, m\right)=\frac{2 i k_{y}^{2}}{k_{0}^{L}} \sum_{n=0}^{\infty} \frac{\left(B_{z O}-(-I)^{n_{1}} B_{z L}\right) \cos k_{n} x}{\left(k_{n}^{2}+k_{y}^{2}\right) \varepsilon\left(\vec{k}_{n}, \omega\right)} \\
& -\frac{2 i k_{0}}{L} \sum_{n=1}^{\infty} \frac{k_{n}^{2}\left(b_{z o}-(-1)^{n} b_{z L}\right) \cos k_{n} x}{\left(k_{n}^{2}+k_{y}^{2}\right)\left(k_{n}^{2}-k_{p}^{2}\right)}
\end{aligned}
$$

The second infinite series in the expression for $\mathbb{E}_{\mathrm{y}}$ can be summed so that

$$
\begin{aligned}
E_{y}\left(x ; k_{y}, \omega\right) & =\frac{2 i k_{y}^{2}}{k_{0}^{L}} \sum_{n=0}^{\infty} \frac{\left(B_{z 0}-(-I)^{n_{B}} B_{z}\right) \cos k_{n} x}{\left(k_{n}^{2}+k_{y}^{2}\right) \varepsilon\left(\vec{k}_{n}, \omega\right)} \\
& -\frac{i k_{y}}{k_{0}} \frac{B_{z 0} \cosh k_{y}(L-x)-B_{z L} \cosh k_{y} x}{\left(I-\omega_{p}^{2} / \omega^{2}\right) \sinh k_{y} I} \\
& +\frac{i k_{p}}{k_{0}} \frac{B_{z 0} \cos k_{p}(L-x)-B_{z L} \cos k_{p} x}{\left(I-\omega_{p}^{2} / \omega^{2}\right) \sin k_{p} L}
\end{aligned}
$$

The infinite series of terms involving the longitudinal dielectric function $\varepsilon\left(\vec{k}_{n}, w\right)$ represents the field due to the longitudinal plasma waves which are coupled to the electromagnetic fieia polarized 
with $\vec{E}$ in the xy plane. For frequencies close to the plasma frequency these waves are important, and are the main feature of interest here. In the result of the linearized calculation of the self-consistent electric field, the dielectric function $\varepsilon\left(\vec{k}_{n}, \omega\right)$ appears in the denominator. The field is therefore eninanced due to the collective hehavior of the plasma under those conditions for which $\varepsilon\left(\vec{k}_{n}, w\right)$ is smail. The last two terms in equation 2.51 were obtained by summing the infinite series of modes of the "transverse" part of the electric field (the divergence-free part). The characteristic lengths for the variation of these terms are comparable with or greater than the wavelength of clectromagnctic wavco in vacuum. Their effective phase velocities are comparable with or greater than that of light, anä hence much greater than that of the particles in the plasma, so they are not oreatly affected by the thermal motions of the particles.

In summary, we have obtained the self-consistent electric fieid In a homogeneous plasma slab in terms of the boundary values of the tangential components of the magnelic fleld al hit surfacts of the slab. The Laplace transforms with respect to time and Fourier transforms with respect to the $y$ and $z$ coordinates (with $k_{z}=0$ ) are given by equations $2.46,2.49$ and 2.21 . 'l'he dependence of the solution on the initial conditions has been discarded; orly the part of the current density proportional to the field disturbance $E_{1}$ has been kept, and this has been assumed to be a small perturbation to the particle motions. 


\section{COLLISIONLESS ABSORPTION IN A PLASMA SLAB}

\section{A. Surface Impedance}

In the previous section the solution to a boundary value problem was obtained, giving the eiectric fiela in a plasma siab in terms of the houndary values of the tangentiai magnetic field. Tn practical applications these boundary values are unknown, however, and must be soived for. The procedure which must be used is to assume the form of the field colution outside the plasma, and match it to the solution inside the plasma by requiring that the tangential electric and magnetic fields be continuous at the boundary. To avoid carrying an unimportant multiplicative conotant in thio procodurc, it is convenient to match the ratio of field components, e.g., $\mathrm{E}_{\mathrm{y}} / \mathrm{B}_{\mathrm{z}}$, at the boundary. The parameters in the field solution outside the plasma can then be related to the properties of the plasma.

Suppose we assume the fields outside the plasma to be those appropriate to a piane, monochromatic wave incident on the plasma at angle $\theta$, polarized with the electric vector in the plane of inclaence (the $x y$ plane), with corresponaing reflected and transmitted waves. Strictiy speaking, the amplitudes of these plane waves should be regaraed as Fourier transforms with respect to $\mathrm{y}$ and $\mathrm{z}$, and Laplace transforms with respect to time, of arbitrary fields satisfying Maxwell's equations in vacuum. To insure convergence of the integral defining the Laplace transform, it is necessary to require that Im $w>0$. The results for a nearly monochromatic wave (not exactly monochromatic because it was turned on at some time in the past) can 
be obtained at the end of the calculation by passing to the limit $(\operatorname{Im} \omega) \rightarrow 0$

The fields of the incident and reflected waves on one side of the slab, and the transmitted wave on the other side, may be written as follows (except for an unimportant multiplicative factor and the factor $\left.e^{i\left(k_{y} y-\omega t\right)}\right)$ :

$$
\begin{aligned}
& E_{y}=\left\{\begin{array}{l}
\left(e^{i k x}-R e^{-i k x}\right) \cos \theta, x \leq 0 \\
T e^{i k x(x-L)} \cos \theta, x \geq L
\end{array}\right\} \\
& B_{z}=\left\{\begin{array}{l}
e^{i k_{x} x}+R e^{-i k_{x} x}, x \leq 0 \\
T e^{i k_{x}(x-I)}, x \geq L
\end{array}\right\}
\end{aligned}
$$

where $k_{y}=k_{0} \sin \theta, k_{x}=k_{0} \cos \theta$.

The ratio $\mathrm{E}_{\mathrm{y}} / \mathrm{B}_{\mathrm{z}}$ at the boundary $\mathrm{x}=\mathrm{I}$, is equal to $\cos \theta$, with the assumed field solution for $x \geqslant L$. Using this boundary condition the ratio $\mathrm{E}_{\mathrm{y}} / \mathrm{B}_{\mathrm{z}}$ at the boundary $\mathrm{x}=0$ can be determined from the field solution in the plasma slab. Denoting this ratio by $Z$, the surface impedance, the continuity condition at $x=0$ takes the form

$$
\frac{(1-R) \cos \theta}{1+R}=\mathrm{Z} \text {. }
$$

Hence, the reflection coefficient $R$ is given in terms of the plasma properties (contained in the surface impedance) by

$$
R=\frac{1-Z / \cos \theta}{1+2 / \cos \theta}
$$

Although the above procedure is fairly simple in principle, it can become algebraically complicatcd. An alternate procedure can be 
usea, which takes advantage of the symmetry of the slab with respect to reflection through its midplane $x=\mathrm{L} / 2$. In this procedure we consider plane waves incident on the plasma slab from both sides, with the same amplituãe and angle of incidence, so that the ratio $\left|E_{y}\right| /\left|B_{z}\right|$ is the same at both bounararies. If the phase and polarization $(\vec{E}$ in the plane of incidence) are specified for one wave, there are two possibilities for the other wave. We call the resulting field configurations "symmetric", or "antisymmetric", accordine to whether the ratio $\mathrm{E}_{\mathrm{yo}} / \mathrm{E}_{\mathrm{yL}}$ is $+I$ or $-I$. In the symmetric case the fields may be written as follows:

$$
\begin{aligned}
& E_{y}^{(s)}=\left\{\begin{array}{l}
\frac{I}{2}\left(e^{i k_{x} x}-R_{s} e^{-i k_{x} x}\right) \cos \theta, x \leq 0 \\
\frac{1}{2}\left(e^{i k_{x}(L-x)}-R_{s} e^{-i k_{x}(L-x)}\right) \cos \theta, x \geq L
\end{array}\right\} \\
& B_{z}^{(s)}=\left\{\begin{array}{l}
\frac{I}{2}\left(e^{i k_{x} x}+R_{s} e^{-i k_{x} x}\right), x \leq 0 \\
-\frac{I}{2}\left(e^{i k_{X}(L-x)}+R_{s} e^{-i k_{X}(L-x)}\right), x \pm L
\end{array}\right\}
\end{aligned}
$$

The amplitude $R_{s}$ of the scattered wave relative to the incident wave is the same on both. sides of the slab, by symmetry. It is related to the surface impedance $z_{s}$ defined by

$$
z_{s}=\left[E_{y}^{(s)} / B_{z}^{(s)}\right]_{X=0}=-\left[E_{y}^{(s)} / B_{z}^{(s)}\right]_{x=L}
$$

as follows : 


$$
R_{s}=\frac{1-z_{s} / \cos \theta}{1+z_{s} / \cos \theta} .
$$

In the antisymmetric case the fields may be written

$$
\begin{aligned}
& \mathrm{E}_{\mathrm{y}}^{(a)}=\left\{\begin{array}{l}
\frac{1}{2}\left(e^{i k_{x} x}-R_{a} e^{-i k_{x} x}\right) \cos \theta, x=0 \\
-\frac{1}{2}\left(e^{i k_{x}(L-x)}-R_{a} e^{-i k_{x}(L-x)}\right) \cos \theta, x=L
\end{array}\right\} \\
& B_{z}^{(a)}=\left\{\begin{array}{l}
\frac{1}{2}\left(e^{i k_{x} x}+R_{a} e^{-i k_{x} x}\right), x \leq 0 \\
\frac{1}{2}\left(e^{i k_{x}(L-x)}+R_{a} e^{-i k_{x}(L-x)}\right), x \geq I
\end{array}\right\} .
\end{aligned}
$$

The scattering coefficient, $R_{a}$ is related to the surface imperance $z_{a}$, defined by

$$
Z_{a}=\left[E_{y}^{(a)} / B_{z}^{(a)}\right]_{x=0}=-\left[E_{y}^{(a)} / B_{z}^{(a)}\right]_{x=I}
$$

as follows:

$$
R_{a}=\frac{I-z_{a} / \cos \theta}{I+z_{a} / \cos \theta} \text {. }
$$

The fields in these symmetric and antisymmetric cases are the same as the symmetric and antisymmetric parts of the unsymmetric fields 3.1 and 3.2 :

$$
E_{y}^{(s)}(x)=\frac{1}{2}\left[E_{y}(x)+E_{y}(L-x)\right], \quad E_{y}^{(a)}(x)=\frac{1}{2}\left[E_{y}(x)-E_{y}(L-x)\right]
$$

(and similarly for $B_{z}$ ) provided we make the identifications $R_{s}=R-T, R_{a}=R+T$. That is, the fields for the unsymmetric case, 
equations 3.1 and 3.2 , can be considered as the superposition of the symmetric and antisymmetric fields:

$$
E_{y}=E_{y}^{(a)}+E_{y}^{(s)}, \quad B_{z}=B_{z}^{(a)}+B_{z}^{(s)} \text { (see Figure 1) }
$$

where

$$
R=\frac{1}{2}\left(R_{a}+R_{S}\right) \quad \text { and } \quad T=\frac{1}{2}\left(R_{a}-R_{s}\right)
$$

Hence, the scattering coefficients $R$ and $T$ are related to $z_{a}$ and $z_{s}$ by equations $3.10,3.9$ and 3.6 .

The surface impedances $Z_{a}$ and $Z_{s}$ for a homogeneous plasma slab can be obtained from the solution for the field in the plasma, given by equation 2.5I. In the "symmetric" case we have $B_{z I}=-B_{z 0}$, so we obtain, by setting $x=0$ in 2.51:

$$
\begin{aligned}
z_{s}=\frac{4 i k_{y}^{2}}{k_{0}^{L}} \sum_{n \text { even }}^{\prime} & \frac{1}{\left(k_{n}^{2}+k_{y}^{2}\right) \varepsilon\left(\bar{k}_{n}, \omega\right)}-\frac{i k_{y}}{k_{0}}\left[\frac{\operatorname{cosin} k_{y} L+1}{\left(1-\omega_{p}^{2} / \omega^{2}\right) \sinh k_{y} L}\right] \\
& +\frac{i k_{p}}{k_{0}}\left[\frac{\cos k_{p} L+1}{\left(1-\omega_{p}^{2} / \omega^{2}\right) \sin k_{p} L}\right]
\end{aligned}
$$

Dimilarly, in the "antioymmetric" cace wo have $\mathrm{B}_{\mathrm{zL}}=\mathrm{B}_{\mathrm{z}}$, so

$$
\begin{gathered}
z_{a}=\frac{4 i k_{y}^{2}}{k_{0}^{L}} \sum_{n \text { odd }} \frac{1}{\left(k_{n}^{2}+k_{y}^{2}\right) \varepsilon\left(\vec{k}_{n}, \omega\right)}-\frac{i k_{y}}{k_{0}}\left[\frac{\cosh k_{y} L-1}{\left(1-\omega_{p}^{2} / \omega^{2}\right) \sinh k_{y} L}\right] \\
+\frac{i k_{p}}{k_{0}}\left[\frac{\cos k_{p} L-1}{\left(1-\omega_{p}^{2} / \omega^{2}\right) \sin k_{p} L}\right]
\end{gathered}
$$



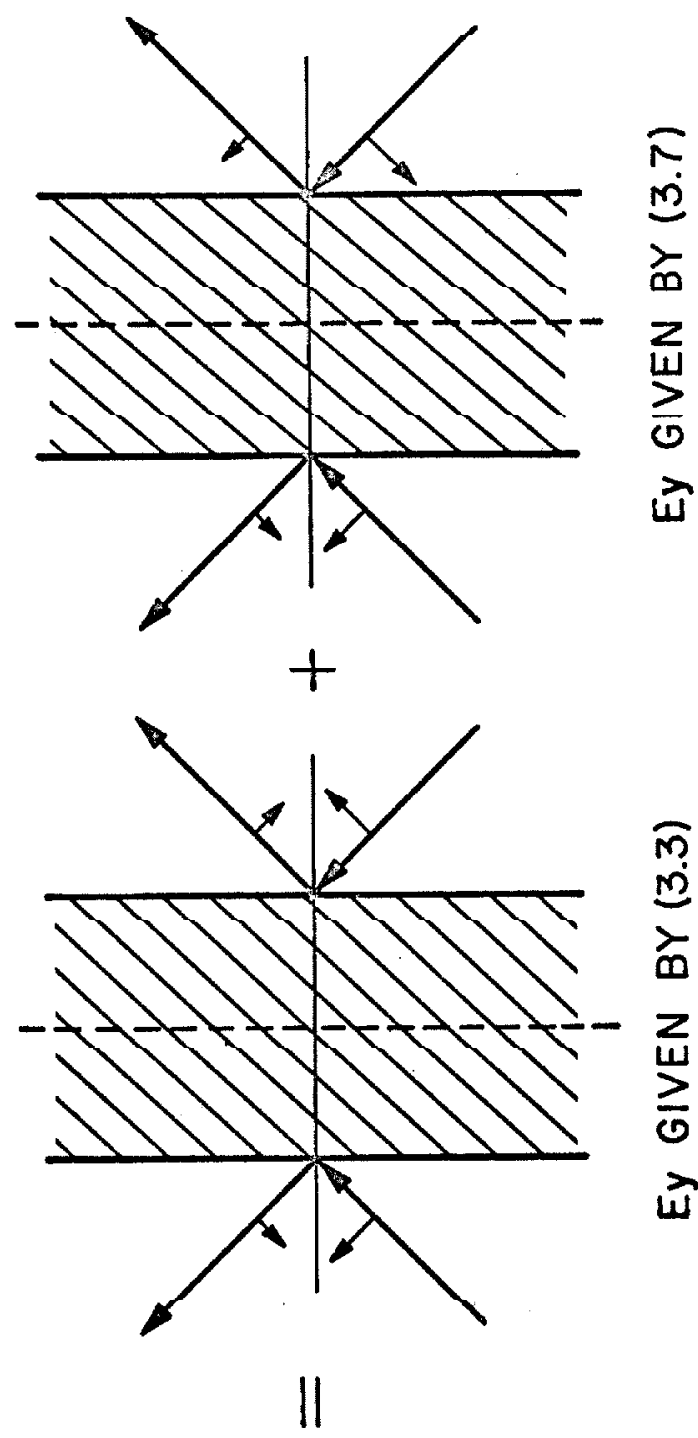

$n$

M

خั

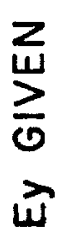

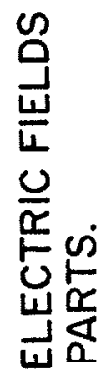

盛足

z음늠

흘

$0 \gtreqless$

这衣

80

w

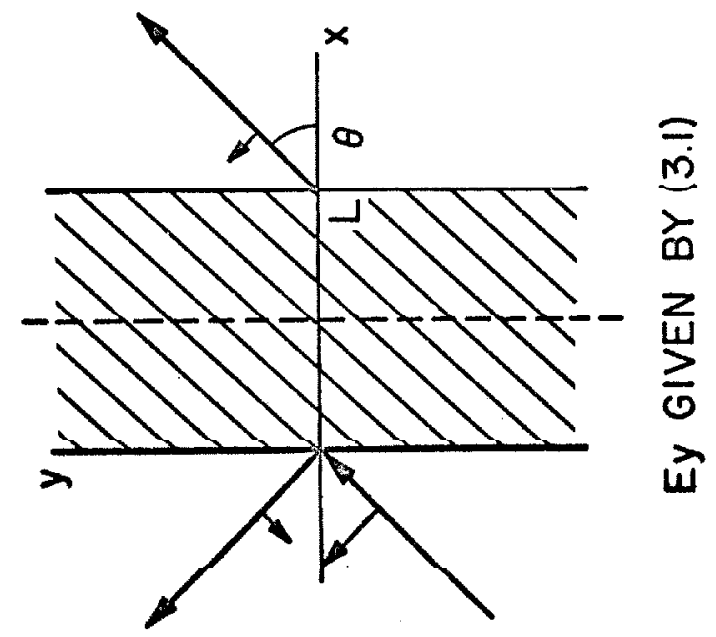

范 
In equations 3.11 and $3.12, k_{p}=k_{0} \sqrt{\cos ^{2} \theta-\omega_{p}^{2} / \omega^{2}}$, and $\mathrm{k}_{\mathrm{y}}=\mathrm{k}_{\mathrm{o}} \sin \theta$.

The last two terms in each of equations 3.11 and 3.12 appear to become infinite for $\omega=\omega_{p}$, but the infinite parts of the two terms cancel. That is, the sum of the last two terms has a finite limit for $w \rightarrow w_{p}$. It will be sufficient in the following to approximate equations 3.11 and 3.12 by replacing the last two terms by their limiting values for $\omega \rightarrow \omega_{p}$ :

$$
\begin{aligned}
& z_{s} \simeq \frac{4 i k_{y}^{2}}{k_{0} L} \sum_{n \text { even }}^{\prime} \frac{1}{\left(k_{n}^{2}+k_{y}^{2}\right) \in\left(\vec{k}_{n},(1)\right.} \\
& -\frac{i\left(k_{o} L\right)\left(\cosh k_{y} L+1\right)\left(\sinh k_{y} L / k y L-I\right)}{2 \sinh ^{2} / k_{y} L} \\
& z_{a} \simeq \frac{4 i k_{y}^{2}}{k_{o}^{L}} \sum_{n \text { odd }} \frac{1}{\left(k_{\mu}^{2}+k_{y}^{2}\right) \varepsilon\left(\vec{k}_{n,}, w\right)} \\
& -\frac{i\left(k_{0} L\right)\left(\cosh k_{y} L-I\right)\left(\sinh k_{y} L / k_{y} L+I\right)}{2 \sinh ^{2} k_{y} L}
\end{aligned}
$$

These approximations are valid for frequencies close to the plasma frequency, which is the case of most interest. In the $n=0$ term in equation 3.13 , the dielectric function is real, $\varepsilon\left(\hat{\mathrm{y}}_{\mathrm{y}}, \omega\right) \simeq$ 1. $\omega_{p}^{2} / \omega^{2}$, since $f_{0}(\vec{u})=0$ for $u_{y}=c / \sin \theta$ in 2.36 ; no partictes can travel faster than light. 


\section{B. The Absorption Coefficient of a Collisionless Plasma Slab}

The absorption coefficient $\alpha$ of a plasma slab is defined as the ratio of power absorbed to power flow into the slab in the incident wave. For the fields given by equations 3.1 and 3.2 the fraction of

the incident power which is reflected is $|R|^{2}$, and the fraction transmitted is $|T|^{2}$, so the fraction absorbed is $\alpha-1-|\Omega|^{2}-|\Gamma|^{2}$. Using equation 3.10 this can be written as

$$
\alpha=I-\frac{1}{2}\left|R_{s}\right|^{2}-\frac{1}{2}\left|R_{a}\right|^{2}
$$

where $R_{s}$ and $R_{a}$ are the reflection coefficients for the symetric and antisymmetric cases considered in the last section. For these cases there is no power flow across the mldplane of the slab vecause the $\mathrm{x}$-component of the Poynting vector is zero there: $\mathrm{B}_{\mathrm{z}}=0$ in the symmetric case, and $E_{y}=0$ in the antisymmetric case, at $x=L / 2$. Hence, the absorption coerticients in these cases are

$$
\alpha_{s}=1-\left|R_{s}\right|^{2} \quad \text { and } \quad \alpha_{a}=1-\left|R_{a}\right|^{2}
$$

Combining equations 3.15 and 3.16 gives $\alpha=\frac{1}{2}\left(\alpha_{s}+\alpha_{a}\right)$.

Hence, the fraction of power absorbed from the wave is half the sum of the fractions which would be absorbed from the symmetric and antisymmetric parts of the fields separately.

Using equations 3.6 and 3.9 , equations 3.16 may be written 


$$
\alpha_{s}=\frac{4 \operatorname{Re} z_{s} / \cos \theta}{\left|I+z_{s} / \cos \theta\right|^{2}} \quad, \quad a_{a}=\frac{4 R e z_{a} / \cos \theta}{\left|I+z_{a} / \cos \theta\right|^{2}}
$$

which, with 3.17 , gives the absorption coefficient $\alpha$ in terms of the symmetric and antisymmetric surface impedances.

The real parts of the surface impedances which determine the absorption coefficients by equations 3.18 are obtained using the expressions 3.13 and 3.14 for $z_{s}$ and $z_{a}$ :

$$
\begin{aligned}
& \operatorname{Re} z_{s}=\frac{4 k_{y}^{2}}{k_{u}{ }_{n}} \sum_{n-2,4}, \cdots \frac{\operatorname{Im} \varepsilon\left(\vec{k}_{n}, w\right)}{\left|\vec{k}_{n}\right|^{2}\left|\varepsilon\left(\vec{k}_{n}, w\right)\right|^{2}} \\
& \operatorname{Re} z_{a}=\frac{4 k_{y}^{2}}{k_{0} I} \sum_{n=1,3, \cdots} \frac{\operatorname{Im} \varepsilon\left(\vec{k}_{n}, w\right)}{\left|\vec{k}_{n}\right|^{2}\left|\varepsilon\left(\vec{k}_{n}, w\right)\right|^{2}}
\end{aligned}
$$

Using equation 2.36, the explicit formula for the longitudinal dielectric function is

$$
\varepsilon\left(\vec{k}_{n}, \omega\right)=1+\frac{4 \pi i}{\omega} \sigma_{\ell}\left(\vec{k}_{n}, \omega\right)=1-\omega_{p}^{2} \int \frac{d \vec{u} \cdot f_{0}(\vec{u})}{\left(\omega-\vec{k}_{n} \cdot \vec{u}\right)^{2}}
$$

for Im $\omega>0$. Pasoing to the limit $(\operatorname{Im} \omega) \rightarrow 0$, and integrating over the two components of velocity perpendicular to the direction of $\overrightarrow{\mathrm{k}}_{\mathrm{n}}$, we obtain

$$
\operatorname{Re} \varepsilon\left(\vec{k}_{n}, w\right)=1-\frac{\omega_{0}^{2}}{\left|\vec{k}_{n}\right|^{2}} \int_{-\infty}^{\infty} \frac{d v F(v)}{\left(v-w /\left|\vec{k}_{n}\right|\right)^{2}},
$$

and

$$
\operatorname{Im} E\left(\vec{k}_{n}, \omega\right)=-\frac{\pi \omega_{p}^{2}}{\left|\vec{k}_{n}\right|^{2}} \quad F^{\prime}\left(\frac{\omega}{\left|\vec{k}_{n}\right|}\right)
$$


where

$$
F(v)=\int d \vec{u} f_{0}(\vec{u}) \delta\left(v-\frac{\vec{k}_{n} \cdot \vec{u}}{\left|\vec{k}_{n}\right|}\right)
$$

is the one-dimensional velocity distribution, i.e., the function $f_{0}(\vec{u})$ integrated over the two components of velocity perpendicular to $\overrightarrow{\mathrm{k}}_{\mathrm{n}}$. But from the assumed isotropy of $f_{0}(\vec{v}), F(v)$ does not depend upon the direction of $\vec{k}_{n}$. In equation 3.21 the principal part is taken with respect to the pole in the integrand. The integral in equation 3.22 gives the result of integrating around a small semicircle, passing below the pole in the complex $v$ plane. It is proportional to the derivative of the one-dimensional probability density for the component of velocity in the direction of propagation of the $n^{\text {th }}$ normal mode of the slab, evaluated at its phase velocity.

Combining equations $3.28,3.20$ and 3.22 , we have

$$
\alpha_{a}=\frac{\omega \omega_{p}^{2} \sin ^{2} \theta}{c \cos \theta\left|I+z_{a} / \cos \theta\right|^{2}} \frac{16 \pi}{I} \sum_{n=1,3, \cdots} \frac{-F^{\prime}\left(\frac{\omega}{\left|\vec{k}_{n}\right|}\right)}{\left|\vec{k}_{n}\right|^{4}\left|\varepsilon\left(\overrightarrow{\hat{k}}_{n}, \omega\right)\right|^{2}}
$$

$\alpha_{S}$ is given by a similar expression, with $Z_{a}$ replaced by $Z_{S}$, and the summation being over $n=2,4,6, \cdots$. Finaliy, the general expression for the absorption caefficient is given, according to equation 3.17 , as follows:

$$
\alpha=\frac{1}{2}\left(\alpha_{s}+\alpha_{a}\right)=\frac{\omega \omega_{p}^{2} \sin ^{2} \theta}{c \cos \theta} \frac{8 \pi}{L} \sum_{n=1}^{\infty} \frac{-F^{\prime}\left(\frac{\omega}{\left|\vec{k}_{n}\right|}\right)}{\left|1+z_{n} / \cos \theta\right|^{2}\left|\vec{k}_{n}\right|^{1 /}\left|\varepsilon\left(\vec{k}_{n}, \omega\right)\right|^{2}}
$$

where $z_{n}=\left\{\begin{array}{ll}z_{s}, & n \text { even } \\ z_{a}, & n \text { odd }\end{array}\right\}$ 
The absorption coefficient is given as an infinite series, corresponding to the infinite number of normal modes of the plasma slab. The $\mathrm{n}^{\text {th }}$ term determines the damping of the plasma waves excited in the slab by the incident electromagnetic waves in the $\mathrm{n}^{\text {th }}$ mode. The damping of this mode is due to electrons whose velocities happen to be nearly the same as the phase velocity of the mode, and is proportional to the derivative of the velocity distribution at this phase velocity. This type of damping was first discussed by Landau (5) in connection with the initial-value problem for plasma waves in an infinite homogeneous medium. It will be discussed in more detail in connection with the present boundary-value problem in section $D$.

For any given frequency $\omega$ all plasma wave modes will be present in the slab. As indicated by equation 3.24, the damping of all these modes contributes to the power absorption from the external fields. It is interesting to note that particles contribute to the collisionless absorption if they belong to any one of a large number of velocity classes. This is characteristic of bounded or inhomogeneous plasmas.

Each plasma wave mode has a definite wavelength. If the frequency is chosen to be the natural frequency for plasma waves with one of these wavelongths, then that particular mode wi3l he strongly excitea. Thus there is a sequence of frequencies $\left\{\omega_{n}\right\}$ at which the response of the plasma to the incident wave may be quite large, and the power absorption may bc cxpcctca to be corrcopondingly large. These natural frequencies are defined by the condition that 
$\left|\varepsilon\left(\vec{k}_{n}, \omega_{n}\right)\right| \simeq 0$, which is the dispersion relation for longitudinal plasma waves. Althougin Im $\varepsilon$, given by equation 3.22 , camnot be zero in general for $\omega /\left|\vec{k}_{n}\right|$ finite, it can be very small for $\omega /\left|\vec{k}_{n}\right|$ much larger than the electron mean thermal speed. (This assumes that the derivative of the electron velocity distribution function goes to zero reasonably fast for large velocities.)

Assuming $\omega /\left|\vec{k}_{n}\right|$ to be large, the following asymptotic expression for Re $\varepsilon$ can be obtained from equation 3.21:

$$
\operatorname{Re} \varepsilon\left(\vec{k}_{n}, \omega\right) \simeq 1-\frac{\omega_{p}^{2}}{\omega^{2}}\left[1+3 \frac{\left|\vec{k}_{n}\right|^{2}\left\langle v^{2}\right\rangle}{\omega^{2}}\right]
$$

for $\left(\left|\vec{k}_{n}\right|^{2}\left\langle v^{2}\right\rangle / \omega^{2}\right) \ll 1$, where $\left\langle v^{2}\right\rangle=\int_{-\infty}^{\infty} d v v^{2} F(v)$. The frequencies $\left\{\omega_{n}\right\}$ may then be determined approximately by setting $\operatorname{Re} \varepsilon=0$ which gives

$$
\omega_{n}^{2} \simeq \omega_{p}^{2}+3\left|\vec{k}_{n}\right|^{2}\left\langle v^{2}\right\rangle
$$

Assuming that $\operatorname{Im} \varepsilon\left(\overrightarrow{\mathrm{k}}_{n}, \omega_{n}\right) \ll 1$, we have $\left|\varepsilon\left(\overrightarrow{\mathrm{k}}_{n}, \omega_{n}\right)\right| \ll 1$. It follows that the amplituaje of the $n^{\text {th }}$ moae of the electric field $E_{x}$ (equation 2.49), which varies like $\sin k_{n} x$, is quite large at the frequency $\omega=\omega_{n}$. This is pecause of the $\varepsilon\left(\vec{k}_{n}, w\right)$ in the denominator of the $n^{\text {th }}$ term in the first series in equation 2.49. The plasma wave with wavelength corresponding to the $n^{\text {th }}$ mode of the slab has been assumed to be only slightly damped, so that its amplitude may be quite large when it is driven by external fields at its natural frequency. It follows also that the real part of the surface impedance $Z_{s}$ or $Z_{a}$ may be quite large at the frequency $\omega=\omega_{n}$, because of 
the $\left|\varepsilon\left(\vec{k}_{n}, \omega\right)\right|^{2}$ in the denominator of the $n^{\text {th }}$ term of equation 3.19 for $n$ even, or 3.20 for $\mathrm{n}$ odd. The absorption coefficient given by equation 3.24 might also be expected to be large at the frequencies $\left\{\omega_{n}\right\}$, but there are further conditions that this be so, which are discussed in Section $\mathrm{C}$.

The above discussion is based on the assumption that the plasma wave modes have small damping. This can only be true if the mode number $\mathrm{n}$ is not too large. This can be made more precise by considering the important special case of a Maxwellian electron velocity distribution

$$
F(u)=\frac{1}{\pi^{1 / 2} v_{e}} e^{-u^{2} / v_{e}^{2}}
$$

The mean-square velocity in one dirention is

$$
\left\langle u^{2}\right\rangle=\frac{1}{2} v_{e}^{2} \equiv \kappa T e / m
$$

and the natural frequency of the $n$th more of the slah is given hy

$$
\omega_{n}^{2}=\omega_{p}^{2}\left[1+\frac{3}{2 \zeta_{n}^{2}}\right] \text { for } r_{n}^{2}>1 \text {, }
$$

where

$$
r_{n}^{2}=\frac{\omega_{p}^{2}}{\left|\vec{k}_{n}\right|^{2} v_{e}^{2}}=\left[2\left(\frac{n \pi \lambda_{D}}{L}\right)^{2}+\frac{v_{e}^{2}}{c^{2}} \sin ^{2} \theta\right]^{-1}
$$

and $\lambda_{D}=\left(\mathrm{KT}_{\mathrm{e}} / 4 \pi n \mathrm{e}^{2}\right)^{1 / 2}$ is the Debye length. With the wave-vector and frequency of the $n^{\text {th }}$ mode, the 1maginary part of the dielectric function has a value given approximately by 


$$
\operatorname{Im} \varepsilon\left(\overrightarrow{\mathrm{k}}_{\mathrm{n}}, \omega_{\mathrm{n}}\right) \simeq 2 \pi^{1 / 2} \zeta_{n}^{3} \mathrm{e}^{-3 / 2} \mathrm{e}^{-\zeta_{n}^{2}}
$$

Therefore, the $n$th mode will have small damping, i.e. Im $\varepsilon\left(\vec{k}_{n}, \omega_{n}\right) \ll 1$ if $n \ll\left(L / \sqrt{2} \pi \lambda_{D}\right)$. We will assume that the slab thickness is many Debye lengths: $L / \lambda_{D} \gg I$ so that there will be several modes whose damping is small. As a numerical example, if $\mathrm{r}_{2} \prec(0.3)\left(\mathrm{L} / \sqrt{2} \pi \lambda_{D}\right)$, say, then $\operatorname{Im} \varepsilon\left(\overrightarrow{\mathrm{i}}_{n}, \omega_{n}\right) \gtrless 0.04$. For $\left(L / \sqrt{2} \pi \lambda_{D}\right)=20$, this condition is satisfied for $n \approx 6$. Larger values of $n$ correspond to wavelengths comparable to the Debye length, which have more Landau damping. C. Absorption Lines and Scattering Resonances

The behavior of the absorption coefficient, as a function of frequency, is not immediately apparent from equation 3.24. If we go back to equation 3.18 we have

$$
\alpha_{a}=\frac{4 \operatorname{Re} z_{a} / \cos \theta}{\left(I+\operatorname{Re} z_{a} / \cos \theta\right)^{2}+\left(\operatorname{Im} z_{a} / \cos \theta\right)^{2}}
$$

and a similar expression for $\alpha_{s}$. It is true that Re $z_{a}$ may be quite large at the natural frequencies $\left\{\omega_{n}\right\}$ of the plasma wave normal modes, and its frequency behavior may take the form of narrow resonance lines at these frequencies if the wave damping is small (sec Figure 2). But the denominator of equation 3.31 contains $\operatorname{Re} \mathrm{Z}_{\mathrm{a}}$ to a higher power than the numerator, so the behavior of $\alpha_{a}$ near the resonance frequencies is not clear.

To determine the behavior of the absorption coefficient for $w$ in the vicinity of $\omega_{n}$, it is convenient to use the following approximation to the dielectric function: 

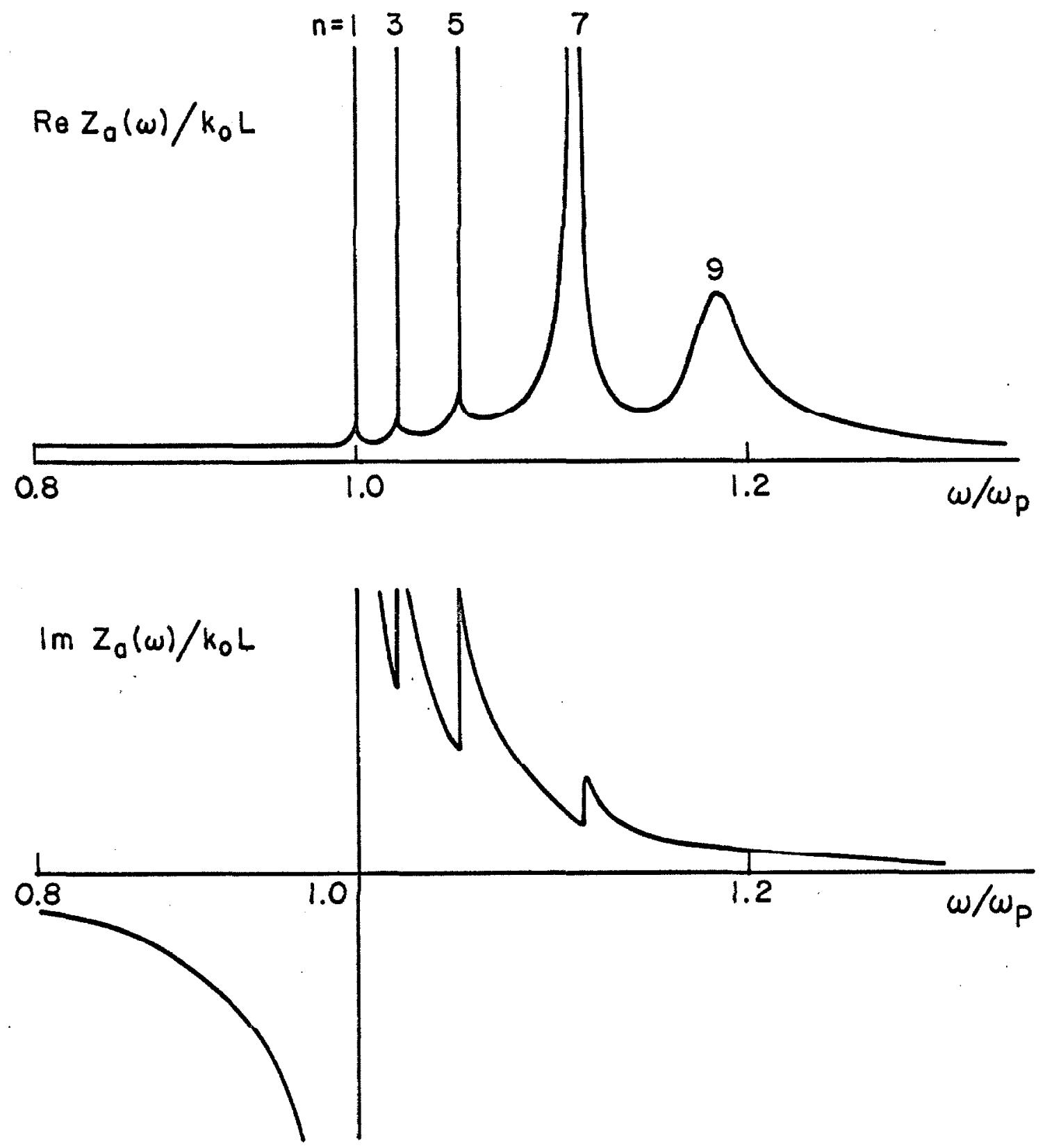

Figure 2: REAL AND IMAGINARY PARTS OF NORMALIZED ANTISYMMETRIC SURFACE IMPEDANCE $Z_{a}(\omega) / k_{0} L$ FOR A "THIN"PLASMA SLAB $\left(k_{0} L \ll 1\right)$.THE VERTICAL SCALE IN THE UPPER CURVE IS MAGNIFIED BY A FACTOR OF ABOUT 45 COMPARED WITH THE LOWER CURVE. SLAB THICKNESS: $L / \sqrt{2} \pi \lambda_{D}=20$. ANGLE OF INCIDENCE: $\theta=45^{\circ}$ [BASED ON NUMERICAL. CALCULATIONS BY R.W. GOULD, FOR THE "PLASMA CAPACITOR" PROBLEM.] 


$$
\varepsilon\left(\vec{k}_{n}, \omega\right) \simeq 2\left[\left(\omega / \omega_{n}-1\right)+i \delta_{n}\right] \text {. }
$$

This is valid for $\omega \simeq \omega_{n}$, provided $\delta_{n} \ll I$, where

$$
\delta_{n}=\frac{1}{2} \operatorname{Im} \varepsilon\left(\vec{k}_{n}, \omega_{n}\right) \equiv \gamma_{n} / \omega_{n}
$$

$\left(\gamma_{n}\right.$ is the Landau damping rate of the $n^{\text {th }}$ mode).

$$
\text { Now using only the } n^{\text {th }} \text { term in equation } 3.14 \text { (where } n \text { is odd) }
$$

we have

$$
\operatorname{Re} z_{a}(\omega) \simeq \frac{4 k_{y}^{2}}{k_{0} I\left(k_{n}^{2}+k_{y}^{2}\right)} \operatorname{Im}\left[\frac{-1}{\varepsilon\left(\vec{k}_{n}, \omega\right)}\right] \text {, for } \omega \simeq \omega_{r_{1}} \text {. }
$$

The other terms in the series can be neglected for $\omega \simeq \omega_{n}$. Using equation 3.32 and setting $k_{y}=k_{0} \sin \theta$, we have

$$
\operatorname{Re} z_{a}(\omega) / \cos \theta \simeq \frac{r_{n} \delta_{n}}{\left(\omega / \omega_{n}-1\right)^{2}+\delta_{n}^{2}} \text {, for } \omega \simeq \omega_{n}
$$

where

$$
r_{n}=\frac{2\left(k_{0} L\right) \sin ^{2} \theta}{\cos \theta\left(n^{2} \pi^{2}+k_{0}^{2} L^{2} \sin ^{2} \theta\right)}
$$

is a measure of the strength of coupling betwecn the ${ }^{\text {th }}$ mode and the electromagnetic field. Equation 3.34 expresses the fact that, near the resonance frequency $\omega_{n}, \operatorname{Re} \mathrm{z}_{\mathrm{a}}(\omega)$ can be approximatea by a narrow Lorentzian, provided the rolative width $\delta_{n}$ is omall comparcd with unity.

Now equations 3.14 and 3.32 may be used to obtain an approximate expression for $\operatorname{Im} z_{\varepsilon}(\omega)$, for $\omega \simeq \omega_{n}$. Let us assume at first, 
that $\mathrm{n}$ is odd but not equal to one, i.e. $\mathrm{n}=3,5, \cdots$. From Figure 2 one sees that woti the first and the $n^{\text {th }}$ terms in the series in equation 3.14 are equally important rumerically. The first term varies relatively slowly with frequency near $\omega_{n}$, so it can be replaced by ils value at $\omega=\omega_{n}$. We fird liat

where

$$
\operatorname{Im} z_{a}(\omega) / \cos \theta=\frac{r_{n}\left(\omega / \omega_{n}-1\right)}{\left(\omega / \omega_{n}-1\right)^{2}+\delta_{n}^{2}}+c_{n} \text {, for } \omega \simeq \omega_{n}
$$

$$
c_{n}=\frac{r_{1}\left(\omega_{n} / \omega_{1}-1\right)}{\left(\omega_{n} / \omega_{1}-1\right)^{2}+\delta_{1}^{2}}-\frac{\left(k_{0} I_{1}\right)\left(\cosh k_{y} I-1\right)\left(\sinh k_{y} L / k_{y} L+1\right)}{2 \cos \theta \sinh ^{2} k_{y} L}(3.37)
$$

The restriction that $n \neq I$ can now be removed. These expressions can also be used for $n=1$ because the first term in the expression for $c_{n}$ io zcro then, as it ohould be.

Now equations 3.34 and 3.36 can be substituted into equation 3.31 to give an approximate expression for $\alpha_{a}$. After some algebra we rind that (for $w=\omega_{n}$ )

$$
\alpha_{a}(\omega)=\frac{4 \delta_{n} r_{n} /\left(1+c_{n}^{2}\right)}{\left(\omega / \omega_{n}-1+\frac{r_{n} c_{n}}{1+c_{n}^{2}}\right)^{2}+\Delta_{n}^{2}}
$$

where $\Delta_{n}=\delta_{n}+\frac{r_{n}}{\left(1+c_{n}^{2}\right)}$.

Thus the absorption coefficient $\alpha_{a}$, as a function of frequency has a Lorentzian line shape near the frequency $\omega_{n}$. But two new features are present in equations 3.38 and 3.39 . First, the relative 
width of the line $\Delta_{n}$ is the sum of the Landau damping width $\delta_{n}$ and a "rauiation daming width" ${ }_{n} /\left(1+c_{n}^{2}\right)$ which is ho energy rauidued by the $n^{\text {th }}$ mode in one period of the wave divided by $4 \pi$ times the energy stored. Second, there is a relative shift of $-r_{n} c_{n} /\left(1+c_{n}^{2}\right)$ in the resonance frequency because of the radiation damping and because of the effective coupling to the transverse modes of the electromagnetic field and (for $n \neq 1$ ) to the $n=1$ mode.

The absorption coeficlent $\alpha_{a}(\omega)$ will be very small for ail frequencies, because $\delta_{\mathrm{n}} \ll 1$, unless $r_{\mathrm{n}} \ll 1$ also. Thus, a sharp line structure in $\operatorname{Re} Z_{a}(w)$ does not lead to a similar structure for $\alpha_{a}(w)$ unless the radiation damping is as small as the Landau damping. This will be true, for a given value of $\theta$, only* if the slab thickness is small compared with the wavelength of the electromagnetic waves: $k_{0} L \ll 1$. In this case $c_{n}^{?}$ and $r_{n} c_{n}$ can be neglected, compared with unity, and we have (for $\omega \simeq \omega_{n}$ )

$$
\alpha_{a}(\omega) \simeq \frac{4 r_{n} \delta_{n}}{\left(\omega / \omega_{n}-I\right)^{2}+\left(\delta_{n}+r_{n}\right)^{2}}, \text { if } k_{0} L \ll I
$$

\footnotetext{
* From equation 3.35 it io clcar that $r_{n}$ ie aiso omall for a thicls $\operatorname{slab}\left(k_{0} I \gg 1\right)$. But in that case the resonance frequencies of the first $\mathbb{N}$ modes are all approximately equal to $\omega_{p}$, where $i \sim k_{0} L$, so these should be considered together, as a single rcoonance. The cffcctive coupling paramotor for thio combincd resonance is about $N$ times the value of $r_{n}$ for a single one, and is of order unity. Hence, the radiation damping connot bo made ar small as the Landau damping, and the absorption is negligible.
} 
The absorption coefficient $\alpha_{a}$ will then consist of the sum of several such narrow lines, centered at the frequencies $\omega_{1}, \omega_{3}, \omega_{5}, \cdots$. For large values of $\mathrm{n}$, the lines will blend into each other when their widths exceed their spacing.

The ahsorption 7 ine given by equation 3.40 has a peak value of $4 r_{n} \delta_{n} /\left(\delta_{n}+r_{n}\right)^{2}$ whose maximum with respect to $r_{n}$ is equal to one, when $r_{n}=\delta_{n}$. For a given value of $n$, i.e. a given line, this maximum can be obtained by changing either $\theta$ or $k_{0} T_{1}$, so that. the radiation damping is matched to the Landau damping for that line. This is the condition for maximum power transfer from the incident wave to the plasma.

Now recall that $\delta_{n}$ increases with $n$, while $r_{n}$ decreases. If $r_{1}=\delta_{1}$, then the peak value of the $n=1$ line will be greater than that of the others, and the peak values will decrease monotonically with $n$. But if the matching condition $r_{n}=\delta_{n}$ is more nearly satisfied for an odd value of $n$ not equal to one, the peak values of the lines will not aecrease monotonically with $\mathrm{n}$. One of the sharp resonances in the real part of the symmetric surface impedance $Z_{s}$ can be analyzed in a similar way, to determine the behavior of $\alpha_{s}$ in the vicinity of one of the symmetric resonance frequencies. The constant term corresponding to $c_{n}$ in equation 3.36 must contain the $n=0$ term in $I m z_{s}$ evaluated at $\omega_{n}(n \neq 0)$, which is proportional to $\left(k_{0} L\right)^{-1}$. The reactive part of $Z_{s}$ thus increases as $k_{0} I$ decreases, so the symmetric absorption coefficient $a_{s}$ cannot be made large enough to be observable by making the slab 
thin. In fact, for a thin $\operatorname{slab}\left(k_{0} L \ll 1\right)$ we have $\operatorname{Re} z_{s} \lesssim 1$, $\operatorname{Im} z_{\mathrm{s}} \gg 1$, su from 3.10 il fulluws lhal $a_{\mathrm{s}} \lll 1$.

Since $a_{s}$ is negligible for a thin slain, we have, from equation 3.17, $\alpha=\frac{1}{2} \alpha_{a}$. This result can be understood by regarding half the power in the incident wave to be carried by the symmetric parts of the fields, and half by the antisymmetric parts. The symmetric parts are not absorbea, so the absorption coefficient $\alpha$ is just half of the fraction of the power absorbed from the antisymmetric parts. In particuiar, since the maximum value of $\alpha_{a}$ is 1 , the maximum value of $\alpha$ is $1 / 2$.

For a thin slab it follows from equation 3.6 that $R_{s} \simeq-1$, because $\operatorname{Re} Z_{0} \lesssim I$, Im $Z_{G} \gg 1$. For symmetric excitation of a thin slab, the magnetic fields of the incident waves tend to cancel, while the tangential electric fields tend to add. This causes the reactive part of $\mathrm{Z}_{\mathrm{s}}$ to be very large, and most of the power is reflected. Using equation 3.10, the fraction of the power reflected in the unsymmetric case, i.e. the reflectivity, is given by

$$
|R|^{2} \simeq\left|\frac{R_{a}-1 l^{2}}{2}\right|^{2} \text {, or }|R|^{2} \simeq \frac{\left|z_{a} / \cos \theta\right|^{2}}{\left|1+z_{a} / \cos \theta\right|^{2}}
$$

using 3.9 .

$$
\text { Using equations } 3.34 \text { ard } 3.36 \text {, the reflectivily for } w=w_{n}
$$
corresponding to the absorption ine 3.40 is found to be

$$
|R(\omega)|^{2} \simeq \frac{r_{n}^{2}}{\left(\omega / \omega_{n}-1\right)^{2}+\left(\delta_{n}+r_{n}\right)^{2}} \text {, if } k_{0} L \ll 1
$$

This represents a scattering resonance with a Lorentzian profile, at 
the same resonance frequency as the corresponding absorption line, and with the same line width. The peak value is $r_{n}^{2} /\left(\delta_{n}+r_{n}\right)^{2}$, which decreases monotonically with increasing $n$. Its value is $1 / 4$ when the radiation damping is matched to the Landau damping, i.e. $r_{n}=\delta_{n}$. The aboorption cocfficient $\alpha$ then has ito maximum value of $1 / 2$ at the center of the iine, so the half of the power in the antisymmetric part of the incident fields is completely absorbed. The other half, in the symmetric part, is scattered by the plasma, symmetrically to either side. Hence one would observe $1 / 4$ of the incident power to be reflected and $I / 4$ to be transmitted.

These scattering resonances are due to the excitation of piasma waves in the slab, at their resonance frequencies. They are accompanied by absorption lines at the same frequencies for the following reason. The ratio of power dissipated to energy stored by a given plasma wave mode is fixed. When one of the modes is excited to a larger amplitude at its resonance frequency, it stores more energy and must therefore absorb correspondingly more power from the incident wave.

\section{Landau Damping in a Bounded Plasma}

In this section we attempt to explain the physical mechanism by which energy is absorbed in a plasma slab. Consider a single normal mode of the slab, with electric field $E_{x}$ proportional to $e^{i y^{y}}$ sin $k_{n} x$. Inserting the time factor $e^{-i \omega t}$ and taking the real part, we may represent the field as

$$
E_{x}=E_{0} \sin k_{n} x \cos \left(\omega t-k_{y} y\right) .
$$


But the wavelength in the $y$ direction is much longer than the distance the particles can travel during one period of the wave, so we may neglect $k y^{y}$ compared to wt. The field can be written as the sum of two traveling waves, with phase velocities equal in magnitude but opposite in direction:

$$
E_{x}=\frac{E_{0}}{2}\left[\sin \left(k_{n} x-\omega t\right)+\sin \left(k_{n} x+\omega t\right)\right]
$$

The particles which are responsible for the damping are, according to equation 3.22, those with velocities about equal to $\omega / k_{n}$. A particle traveling in a certain direction is essentially affected only by the wave traveling in the same direction, i.e. one or the other of the terms in equation 3.42. If its velocity is nearly equal to $\omega / k_{n}$, its urperturbed trajectory keeps in step with one of the traveling waves until it is reflected, with the other until it is reflected again, and with the first until the next reflection, etc. The phase relablons are preserved, on dilernabe reflections frum lhe vuundaries, if the oscillation frequency of the particle $\Omega$ is approximately equal to $\omega / n$, where $\omega$ is the frequency of the incident wave ano $r_{1}$ is the mode number. The mechanism of collisionless damping in a plasma slab can therefore be discussed in much the same way as for an infinite plasma. The theory of this damping in an infinite plasma

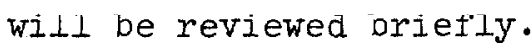

If there is a sinusoidai disturbance in the electron density at some time, then there will be a coherent wave propagating through the plasma at later times. The linearized theory (5) predicts that the wave will be damped, and its energy is transferred to those 
particles with nearly the same velocity as the wave. Some of the particles are accelerated by the electric field of the wave, and some are decelerated, according to their positions relative to the field maxima and minima in the wave. This causes a slow rearrangement, or flow, of the particles in phase space (14).

Because there are more particies traveling slightly slower than the wave than are traveling slightly faster, this flow in phase space results in a net transfer of energy to the resonant particles.

Of course, this can only be true for a time which is short compared to the "trapping time". The periods of oscillation of the resonant particles which are trapped in the potential energy minima in a coordinate system moving with the wave, are greater than or equal to the "trapping time",

$$
\tau_{t r}=\left(\frac{m}{e \varepsilon_{n} k}\right)^{1 / 2}
$$

where $\varepsilon_{n}$ is the amplitude of the wave. For times comparable to $\tau_{t r}$, the net effect of the wave on the resonant particles cannot be considered a small perturbation, and the linearized theory becomes invalid (14).

If close-range encounters with other particles are frequent enough, however, the electrons will be scattered out of the potential wells of the wave before the Iinearized theory becomes invalid. Then the initial-value problem for the motion of a particle may be considered to be started over again after each scattering encounter. Also, if the cumulative effect of long-range encounters is rapid enough, the energy taken from the wave by the resonant electrons during the 
first half of their oscillation periods in the potential wells, will be shared with the other electrons before it can be returned coherently to the wave in the second half of their oscillation periods. Thus the presence of some collisions, or dynamical friction, is necessary to make the linearized theory uniformly valid for all times.

In order that the linearized theory be valid in a steady state, where power is supplied by sources external to the plasma, we must assume that the mean collision rate is much larger than the "trapping rate", or $\bar{v} \tau_{t r} \gg 1$. This places an upper limit on the amplitudes of the normal modes in the plasma, in order that the linearized theory of power ahsorption he valid.

It should be clear from this discussion that the so-called "collisionless" absorption mechanism is really an indirect mechanism by which the plasma absorbs energy from an incident electromagnetic wave and converts it into heat. It is indirect because the energy is first transferred to groups of resonant particles and then to the plasma as a whole. It can be much more efficient than the direct collisional mechanism when longitudinal waves are excited in the plasma, especialiy if there are appreciable numbers of particles with nearly the same velocities as the longituainal waves.

If the nearly collisionless theory is to provide a good description of the damping, the Landau damping rate must be much larger than the mean collision rate. We must. therefore assume the following double inequality to hold: $\quad Y_{n} \gg \bar{v} \gg \tau_{t r}^{-1}$. The effects of collisions will be discussed further in section IVD. 
$-56-$

IV. COLLISIONLESS NOISE RADIATION FROM A PLASMA SLAB

\section{A. Surface Emissivity}

A bounded plasma contains charged particles in motion, which are accelerated by their interactions with each other and with the confining fields and which therefore emit electromagnetic radiation. The radiation fields of different electrons have no definite phase relations between them, so the total radiation field of the plasma has a noise-like character. Although the radiation intensity can be obtained from Kirchhoff's law if the plasma is in thermal equilibrium (this will be done in Section $C$ ), we are concerned here with the more general case.

The radiation fields are determined by the current density in the plasma, which is due to the motions of the charged particles, and these in turn depend upon the initial conditions of all of the particles at some instant of time. But the initial conditions for this extremely large number of particles are unknown. The best that can be aone is to assume the joint probability distribution of these initial conditions to be known. Then the statistical properties of the radiation field can be determined by averaging over the initial conditions of the particles. This procedure will be carried out in Section B.

In this section the relation between the surface emissivity of a plasma slab and the Laplace transforms of the radiation field and the field at the plasma boundary will be derived.

A quantity which might be measured in an idealized experiment is the surface emissivity $I(\theta, w)$. Let. $I(\theta, w)$ d $d w$ be defined 
as the power radiated in a direction making an angle $\theta$ with the slab normal, within solid angle $d \Omega$, in frequency interval $d \omega$, per unit surface area of the slab. Only the single polarization with electric vector in the plane of the radiation direction and the slab normal is counted, because it is the only one which is coupled to the longitudinal fielảs in the plasma. The emissivity is closely related to the spectral flux density of the radiation field, which will now be defined. The Fuynling vector of the electromagnetic field outside the plasma is

$$
\vec{S}(\vec{r}, t)=\frac{e}{4 \pi} \delta \vec{E}(\vec{r}, t) \times \delta \vec{B}(\vec{r}, t)
$$

where the notation for the electric and magnetic fieids is meant to emphasize that these are fluctuation quantities having zero expectation values. The expected value of the component of the Poynting vector in the direction $\hat{r}$ (per $\mathrm{cm}^{2}$ of surface area) is

$$
\begin{aligned}
\frac{1}{A}\langle\hat{r} \cdot \vec{s}(\vec{r}, l)\rangle-\frac{c}{4 \pi A}\langle\hat{r} \cdot \delta \vec{E}(\vec{r}, t) \times s \vec{B}(\vec{r}, t)\rangle & -\int_{0}^{\infty} d \omega s(\omega)
\end{aligned}
$$

which defines the spectral flux density $S(\omega)$. The total surface area of the plasma $\mathrm{slab}$ is $\mathrm{A}=\mathrm{V} / \mathrm{L}$. where $\mathrm{V}$ is the volume and $\mathrm{I}$ the width. (The limit $V \rightarrow \infty$ will be taken later.)

Suppose the direction $\hat{r}$ makes an angle $\theta$ with the slab normal, and the fielas $\delta \overrightarrow{\mathrm{E}}, \delta \overrightarrow{\mathrm{B}}$ are radiation fields propagating ir the direction $\hat{r}$, with the polarization of interest. Then $S(\omega) d \Sigma d \omega$ is the power radiated in frequency interval dw in a direction making an angle $\theta$ with the slab normal which crosses an area $d \Sigma$ at the 
observation point $\vec{r}$ with its normal in the direction of propagation $\hat{r}$ per unit eurface area of the slab. Because $a \Sigma=r^{2} a \Omega$, where $d \Omega$ is the solid angie subtended by the area $d \Sigma$ at a distance $r$, the relation between the surface emissivity and the spectral flux density is

$$
J=r^{2} S(\omega)
$$

The spectral flux density is (except for a constant factor) the Fourier transform of the radiation field autocorrelation function. Hence,

$$
J=r^{2} S(\omega)=\left(\frac{c L r^{2}}{4 \pi^{2} V}\right) \int_{-\infty}^{\infty} d \tau e^{i \omega \tau}\langle\delta E(\vec{r}, t) \delta E(\vec{r}, t+\tau)\rangle
$$

Now $J$ can also be obtained airectly from the Laplace transform of the radiation field as a function of time:

$$
J=\left(\frac{c L r^{2}}{4 \pi^{2} V}\right) \lim 2 \gamma\langle\delta E(\vec{r},-\omega+1 \gamma) \delta E\langle\vec{r}, \omega+i \gamma)\rangle
$$

where $\delta E(\vec{r}, \omega)=\int_{0}^{\infty} d t e^{i \omega t} \delta E(\vec{r}, t)$.

To prove 4.4, substitute the definition 4.5 of the Laplace transform into the right-hand side of equation 4.4. After interchanging the order of integrations over time and the statistical average, we have

$$
\begin{aligned}
& \left\langle\delta E\left(\vec{r},-\omega+i_{\gamma}\right) \quad \delta E\left(\vec{r}, \omega+i_{\gamma}\right)\right\rangle \\
& =\int_{0}^{\infty} d t e^{i\left(-\omega+i_{\gamma}\right) t} \int_{0}^{\infty} d t^{\prime} e^{i\left(\omega+i_{\gamma}\right) t^{\prime}}\left\langle\delta E(\vec{r}, t) \delta E\left(\vec{r}, t^{\prime}\right)\right\rangle
\end{aligned}
$$


Now we replace the integration variable $t^{\prime}$ by $\tau-t^{\prime}-t$, and change the order of integration in the double integral over $t$ and $\tau$. Then we obtain

$$
\begin{aligned}
& \langle\delta E(\vec{r},-\omega+i \gamma) \delta E(\vec{r}, \omega+i \gamma)\rangle= \\
& \quad \int_{0}^{\infty} d \tau e^{i(\omega+i \gamma) \tau} \int_{0}^{\infty} d t e^{-2 \gamma t}\langle\delta E(\vec{r}, t) \delta E(\vec{r}, t+\tau)\rangle \\
& +\int_{-\infty}^{0} d \tau e^{i(\omega+i \gamma) \tau} \int_{-\tau}^{\infty} d t e^{-2 \gamma t}\langle\delta E(\vec{r}, t) \delta E(\vec{r}, t+\tau)\rangle .
\end{aligned}
$$

Assuming that the radiation field is a stationary random function, the autocorrelation function is a function of the time difference only, i.e. $\langle\delta E(\vec{r}, t) \delta E(\vec{r}, t+\tau)\rangle$ is a function of $\tau$ only (15). The integrals over $t$ can therefore be carried out, with the result that

$$
\begin{aligned}
& \langle\delta E(\vec{r},-\omega+i \gamma) \delta E(\vec{r}, \omega+i \gamma)\rangle= \\
& \quad \frac{I}{2 \gamma} \int_{-\infty}^{\infty} a \tau e^{i \omega \tau} e^{-\gamma|\tau|}\langle\delta E(\vec{r}, t) \delta E(\vec{r}, t+\tau)\rangle
\end{aligned}
$$

Hence

$$
\lim _{\gamma \rightarrow 0} 2 \gamma\langle\delta E(\vec{r},-\omega+i \gamma) \delta E(\vec{r}, \omega+i \gamma)\rangle=\int_{-\infty}^{\infty} d \tau e^{i \omega \tau}\langle\delta E(\vec{r}, t) \delta E(\vec{r}, t+\tau)\rangle,
$$

and using 4.3 wo obtain the roquired relation, 1.11.

The surface emissivity can now be obtained from equation 4.4 if the Laplace transform of the radiation field is given. This can be related to the Laplace transform of the field fluctuation $\delta E_{y}$ at the slab boundary, which in turn is related to the plasma properties 
tinrough the solution of the boundary value problem for the field fluctuations. If we are interested in the radiation field in the region $x<0$, i.e., to the left of the slab, we first Fourier analyze the field $\delta E_{\mathrm{y}}(\overrightarrow{\mathrm{r}},(1))$ at the boundary $\mathrm{x}=0$ :

$$
\delta E_{y}(U, y, z, w)=\int_{-\infty}^{\infty} \frac{d k y}{2 \pi} \int_{-\infty}^{\infty} \frac{d k}{2 \pi} e^{i\left(k_{y} k+k z z\right)} \delta F_{y o}\left(k, y, k_{z}, w\right) .
$$

These field fluctuations must now be "analytically continued" away from the surface of the plasma.

The radiation field in vacuum which is a solution of

$$
\left(\nabla^{2}+k_{0}^{2}\right) \delta E_{y}(\vec{r}, \omega)=0 \text {, for } x<0 \text {, }
$$

which has the boundary values given by equation 4.6 at $x=0$, and which represents waves traveling away from the plasma for $x \rightarrow-\infty$, is given by

$$
\delta E_{y}(\vec{r}, \omega)=\int_{-\infty}^{\infty} \frac{d k}{2 \pi} \int_{-\infty}^{\infty} \frac{d k_{z}}{2 \pi} e^{i k_{0} r f\left(k_{y}, k_{z}\right)} \delta E_{y o}\left(k_{y}, k_{z}, \omega\right)
$$

where

$$
f\left(k_{y}, k_{z}\right)=\frac{k_{x} x+k_{y} y}{k_{0} r}=\frac{k_{y}}{k_{0}} \sin \theta+\sqrt{I-\left(k_{y} / k_{0}\right)^{2}-\left(k_{z} / k_{0}\right)^{2}} \cos \theta .
$$

The observation point $\vec{r}$ has been chosen to be in the $x-y$ plane at a distance $r$ from the origin: $\vec{r}=(-r \cos \theta, r \sin \theta, 0)$. If we assume this distance is many wavelengths away from the origin, so that $k_{0} r \gg 1$, then the radiation field $\delta E_{y}$ given by equation 4.7 can be approximated by a spherical wave propagating out from the origin.

To show this we evaluate the double integral in 4.7 approximately, using the stationary phase approximation. Recause of the exponential 
factor in the integrand, which oscillates rapidly as a function of $k y$ and $k_{z}$, there is no significant contribution from most of the region of integration. Only the region near the stationary phase point $\left(k_{y s}, k_{z s}\right)$ where $\partial f / \partial k_{y}=\partial f / \partial k_{z}=0$ needs to be considered, because the exponential factor oscillates most slowly in that region. Assuming that $\delta E_{y o}\left(k_{y}, k_{z}, w\right)$ is a slowly varying function of $k_{y}$ and $k_{z}$, it can be repiaced by its value at the stationary phase point. The exponential factor can be integrated approximately by expanding $f\left(k_{y}, k_{z}\right)$ in a Taylor series about the stationary phase point, which is found to be $\mathrm{k}_{\mathrm{ys}}=\mathrm{k}_{\mathrm{o}} \sin \theta, \mathrm{k}_{\mathrm{zs}}=0$. The stationary phase approximation to the double integral in 4.7 is found to be

$$
\delta E_{y}(\vec{r}, \omega) \simeq \frac{-i k_{0}}{2 \pi} \cos \theta \frac{e^{i k_{0} r}}{r} \delta E_{y o}\left(x_{0} \sin \theta, \omega\right) \text {, for } k_{0} r>1 \text { (4.8) }
$$

where the argument $\mathrm{k}_{\mathrm{z}}=0$ is to be understood. This ropresents a spherical wave going out from the origin whose amplitude is proportional to the Fourier coefficient of the plane wave component in 4.7 which propagates in the direction from the origin to the observation point $\overrightarrow{\mathrm{r}}$.

Thus result can be expressea in terms of $\delta E(\vec{r}, \omega)$, the component perpendicular to the airection of propagation, since $\delta E_{y}=S E$ cus $\theta$. By substituting the result into equation 4.4 , the surface emissivity can be obtained from the field fluctuations at the plasma boundary, as follows: 


$$
J(\theta, \omega)=\left(\frac{\mathrm{ck}_{0}^{2} L}{16 \pi}\right) \frac{1}{V} \lim _{\gamma \rightarrow 0} 2 \gamma\left\langle\delta E_{y o}\left(-k_{0} \sin \theta,-\omega+i \gamma\right) \delta E_{y o}\left(k_{0} \sin \theta, \omega+i \gamma\right)\right\rangle
$$

B. Electric Field Fluctuations in a Plasma Slab

Since the emissivity of a plasma slab is determined by the boundary values of the field fluctuation $\delta \mathrm{E}_{\mathrm{y}}$, it is necessary to determine these boundary values in terms of the plasma properties. This can be done by using the solution of the boundary value problem which was derived in Part II. Although we interpreted all of the fields as ensembie average, or macroscopic fields, an alternate interpretation is possible and is necessary to find the field fluctuations. In the perturbation analysis of the particle equations of motion (Section II.B), $\overrightarrow{\mathrm{E}}_{1}$ is now to be interpretea as the fluctuation of the field from its ensemble average value $\overrightarrow{\mathrm{E}}_{0} \equiv\langle\overrightarrow{\mathrm{E}}\rangle$ :

$$
\delta \vec{E} \equiv \vec{E}-\langle\vec{E}\rangle
$$

No macroscopic disturbances, such as the plasma waves considered in Sections II and III, are to be present.

The current density calculated in Section II.C is then due to particles moving nearly on their unperturbed trajectories in the confining field of the slab, being slightly perturbed by the fiela fluctuation $\delta \overrightarrow{\mathrm{E}}$. The "unperturbed part" of the current density should not be averaged as in equation 2.31 , since it represents the source of the field fluctuations: 


$$
\delta \vec{j}^{(0)}(\vec{x}, t)=q \sum_{j} \vec{v}_{j}^{(0)} \delta\left(\vec{x}-\vec{r}_{j}^{(0)}(t)\right) .
$$

The ensemble average can be used, however, for that part of the current dessily fIuclualion $\vec{\partial}$ which is causea by the riela $\overrightarrow{\mathrm{E}}$, with the condition that $\delta \overrightarrow{\mathrm{E}}(\overrightarrow{\mathrm{x}}, \mathrm{t})$ is a given function. This conditional expectation still has a fluctuation nature because $\delta \vec{E}$ is to be determined by solving Maxwell's equaliuns wiln he current density fluctuation $\delta \vec{j}$ as the source. The neglect of higher-order fiuctuation quantities (16) in this procedure is justified if there are many particles per Debye sphere: ${ }_{n} \lambda_{\mathrm{D}}^{3} \gg 1$. The rluctuation $\vec{\delta} \vec{\jmath}$ is then given by equation 2.32 with $j_{\mu}^{(0)}$ replaced oy $\delta j_{\mu}^{(0)}$ and $E_{1 B}$ by $\delta \mathrm{E}_{\beta}$

The part of $\delta \vec{j}$ aue lo the perturbation of the particle trajectories, caused by the field $\delta \overrightarrow{\mathrm{E}}$, represents the linearized response of the plasma to the superposition of the fields of all the individual particles. The response is calculated as hluugh lisese individual particles moved independently on trajectories determined by the confining fields of the slab and their initial conditions. It is the response of a dielectric medium having the average properties of the collection of charged particles which is the plasma, and it tenas to shield the fields of the individual charges.

The fiela fluctuation $\delta \overrightarrow{\mathrm{E}}$ can therefore be thought of as being produced by non-interacting, independent quasi-particles, i.e. the individual charged particles with their associated shielding ciouds. This is the principle of superposition of the fielas of "dressed test particles", first discussed by Hubbard (17). Rostoker showed that the 
use of these statistically independent quasi-particles is equivalent to a correct treatment of particle correlations (18).

After making the other assumptions and approximations discussed in Section II.C, the electric field fluctuations can be solved for, self-consistently as in Section II.D. The result, corresponding to equation 2.51 , is as follows:

$$
\begin{aligned}
& \delta E_{y}\left(x ; k_{y} \omega\right)=\frac{2 i k_{y}^{2}}{k_{0} L} \sum_{n=0}^{\infty} \frac{\cos k_{n} x}{\left|\vec{k}_{n}\right|^{2} \varepsilon\left(\vec{k}_{n}, \omega\right)}\left\{\delta R_{z 0^{-}}(-1)^{n} \delta R_{z L}\right. \\
& \left.-\frac{4 \pi k_{0}}{k_{y}} \delta \rho(0)\left(\vec{k}_{n}, \omega\right)\right\}-\frac{\left(i / k_{o}\right)}{\left(i-\omega_{p}^{2} / \omega{ }^{2}\right)}\left\{k_{y}\left[\frac{\delta B^{\cosh k_{y}(i-x)-\delta B} z L^{\cosh k_{y} x}}{\sinh k_{y} L}\right]\right. \\
& \left.+k_{p}\left[\frac{\delta B_{z o} \cos k_{p} I-\delta B_{z I}}{\sin k_{p} L}\right]\right\}
\end{aligned}
$$

The "unperturbed charge density fluctuations" $\delta \rho_{c}^{(0)}$ cannot be neglected because they are now the source of the field fluctuations. We have neglected $\delta j_{t}^{(0)} / c$ in comparison with $\delta p_{c}^{(0)}$, since the unperturbed particle trajectories have velocities which are much smaller than $c$. This is consistent with the neglect of $\operatorname{Re} \sigma_{t}$ in the approximation 2.40 .

In addition to the fields induced in the piasma by the fields at the boundary, there are the fields induced by the particle discreteness. The screening effect of the plasma is indicated in equation 4.12 , where the charge density of the "bare" particles $\delta \rho_{c}(0)$ is divided by the dielectric function $\varepsilon\left(\vec{k}_{n}, w\right)$ to obtain the charge density of the "dressed" particles. 
The boundary values of the tangential magnetic field are determined by the condition that the fields $\delta \mathrm{E}_{\mathrm{y}}, \delta \mathrm{B}_{\mathrm{z}}$ be continuous at the boundary. These fields must therefore have the same ratio as the fields outside the plasma which represent outgoing plane waves. This is the radiation condition, which takes the form

$$
\delta E_{y 0} / \delta B_{z O}=-\cos \theta \quad, \quad \delta E_{y L} / \delta B_{z L}=\cos \theta
$$

These relations can be subotitutcd into oquation $l_{1.12}$, but $\delta E_{\text {yo }}$ and $\delta E_{y L}$ must still be solved for. By setting $x=0$ and $x=I$ in equation 4.12 , we obtain two simultaneous algebraic equations for $\delta \mathrm{E}_{\text {yo }}$ and $\delta \mathrm{E}_{\mathrm{yL}}$. The solution of these gives

$$
\delta E_{y o}\left(k_{y}, w\right)=\frac{-8 \pi i}{L} \sum_{n=0}^{\infty} \frac{k_{y} \delta \rho_{c}^{(0)}\left(\vec{k}_{n}, w\right)}{\left[1+z_{n} / \cos \theta\right]\left|\vec{k}_{n}\right|^{2} \varepsilon\left(\vec{k}_{n}, w\right)}
$$

where $z_{n}$ is given by equation 3.25. A similar expression is obtained for $\delta E_{y L}$, but with the $n^{\text {th }}$ term in the series multiplied by $(-1)^{n}$. The electric fiela fluctuation $\delta E_{y}$ in the plasma can now be obtained by substituting these expressions, and the relations 4.13 , into equation 4.12.

Equation 4.14 expresses the basic test-particle property of the plasma slab. The electric field fluctuations are due to the discreteness of the individual charges, and are modified by the collective behavior of the plasma, as indicated by the dielectric function $\varepsilon\left(\vec{k}_{n}, w\right)$ in the denominator. They are also modified by the coupling with the radiation field outside the plasma, as indicated by the presence of the surface impedances. The symmetric surface impedance 
$\mathrm{Z}_{\mathrm{S}}$ appears with the symmetric modes of the charge density fluctuation (even $n$ ), ana the antisymmetric surface impedance $z_{a}$ with the antisymmetric modes (odd $\mathrm{n}$ ).

Using equation 4.14 , the emissivity given by equation 4.9 can be written as follows:

$$
\begin{aligned}
J(\theta, \omega) & =\frac{4 \mathrm{ck}_{0}^{4} \sin ^{2} \theta}{\pi^{2} L} \sum_{m=0} \sum_{n=0} \\
& \times \frac{\frac{1}{V} \lim _{\gamma \rightarrow 0} 2 \gamma\left\langle\delta \rho_{c}^{(0)}\left(-\vec{k}_{n},-\omega+i \gamma\right) \delta \rho_{c}^{(0)}\left(\vec{k}_{m}, \omega+i \gamma\right)\right\rangle}{\left(I+z_{n}^{*} / \cos \theta\right)\left(I+z_{m} / \cos \theta\right)\left|\vec{k}_{n}\right|^{2}\left|\vec{k}_{m}\right|^{2} \varepsilon^{*}\left(\vec{k}_{n}, \omega\right) \varepsilon\left(\vec{k}_{m}, \omega\right)}
\end{aligned}
$$

where $\vec{k}_{n}=\left(n \pi / L, k_{0} \sin \theta, 0\right)$. The emissivity is thus finally related to the statistical properties of the unperturbed part of the charge density:

$$
\begin{array}{r}
\delta \rho_{c}^{(0)}\left(\vec{k}_{n}, \omega\right)=\int_{0}^{\infty} d t e^{i \omega t} \int_{-\infty}^{\infty} d y \int_{-\infty}^{\infty} d z e^{-i\left(k_{y} y+k_{z} z\right)} \\
\times \int_{0}^{L} d x \cos k_{n} x \delta \rho(0)(\vec{x}, t)
\end{array}
$$

where $\delta \rho^{(0)}(\vec{x}, t)=q \sum_{j=1}^{N} \delta\left(\vec{x}-\vec{R}\left(t \mid \vec{r}_{j}, \vec{v}_{j}, 0\right)\right)$, and $\vec{R}\left(t \mid \vec{r}_{j}, \vec{v}_{j}, 0\right)$ is the unperturbed trajectory function discussed in Section II.B. Carrying out the indicated integration we have (for $k_{z}-0$ ) 


$$
\delta p_{c}^{(0)}\left(\vec{k}_{n}, \omega\right)=q \sum_{j=1}^{N} e^{-i k_{y} y_{j}}\left[\frac{i\left(\omega-k_{y} v_{y j}\right) \cos k_{n} x_{j}+k_{n} v_{x j} \sin k_{n} x_{j}}{\left(\omega-k_{y} v_{j j}\right)^{2}-k_{n}^{2} v_{x j}^{2}}\right](4.26)
$$

Since the effect of particle correlatione is included in cquation 4.15 through the use of the dielectric function $\varepsilon\left(\vec{k}_{n}, \omega\right)$, the initial conditions $\left\{\vec{r}_{j}, \vec{v}_{j}\right\}$ for all the particles must now be assumed to be independent random variables. Each of the initial positions $\vec{r}_{j}$ is assumed to be uniformly distributed within the plasma volume, so that the probability density for $\vec{r}_{j}$ is constant within the slab and zero outside. The initial velocities are assumed to be identically distributed with probability density $f_{n}\left(v_{x}, v_{y}\right)$. We then find, in the limit $N, V \rightarrow \infty, N / V=n_{e}$, that

$$
\begin{aligned}
& \frac{I}{v}\left\langle\delta \rho_{c}^{(0)}\left(-\vec{k}_{n},-\omega^{\prime}\right) \delta \rho_{c}^{(0)}\left(\vec{k}_{m}, \omega\right)\right\rangle \\
& \quad \rightarrow \frac{n e^{2}}{2} \delta_{n m} \int_{-\infty}^{\infty} d v_{x} \int_{-\infty}^{\infty} d v_{y} f_{0}\left(v_{x}, v_{y}\right)\left\{\frac{\left(\omega^{\prime}-k_{y} v_{y}\right)\left(\omega-k_{y} v_{y}\right)+k_{n}^{2} v_{x}^{2}}{\left[\left(\omega^{\prime}-k_{y} v_{y}\right)^{2}-x_{n}^{2} v_{x}^{2}\right]\left[\left(\omega-k_{y} v_{y}\right)^{2}-k_{n}^{2} v_{x}^{2}\right]}\right\}
\end{aligned}
$$

for $n \neq 0$ with an additional factor of 2 for $n=0$. We now replace $\omega^{\prime}$ by $\omega-i \gamma$ and $\omega$ by $\omega+$ ir . After some algebraic simplification we multiply by $2 \gamma$ and take the limit $\gamma \rightarrow 0$, making use of the formal relation

$$
\frac{2 \gamma}{x^{2}+\gamma^{2}} \rightarrow 2 \pi \delta(x)
$$

The result can be expressed as follows: 


$$
\frac{1}{\bar{v}} \lim _{\gamma \rightarrow 0} 2 \gamma\left\langle\delta \rho_{c}^{(0)}\left(-\vec{k}_{n},-\omega+i \gamma\right) \delta \rho_{c}^{(0)}\left(\vec{k}_{m}, \omega+i \gamma\right)\right\rangle=\frac{\pi e_{e} e^{2}}{\left|\vec{k}_{n}\right|} \delta{ }_{n m} F\left(\frac{\omega}{\left|\vec{k}_{n}\right|}\right)
$$

for $n \neq 0$, with an additional factor of 2 for $n=0$, where $F(u)$ is the one-dimensional velocity distribution.

Combining equations 4.15 and 4.17 , we have the following general expression for the emissivity:

$$
J(\theta, \omega)=\frac{4 n e^{e^{2} \omega^{4} \sin ^{2} \theta}}{\pi L c^{3}} \sum_{n=1}^{\infty} \frac{F\left(\frac{\omega}{\left|\vec{k}_{n}\right|}\right)}{\left|I+z_{n} / \cos \theta\right|^{2}\left|\vec{k}_{n}\right|^{5}\left|\varepsilon\left(\vec{k}_{n}, \omega\right)\right|^{2}}
$$

(The $n=0$ term has been dropped, since $F\left(\frac{c}{\sin \theta}\right)$ must be zero; particles cannot travel at the speed of light or faster.) This is one of the primary results of this investigation. Under some conditions, which are discussed in the next section, the emissivity is large at those frequencies for which the dielectric function $\varepsilon\left(\overrightarrow{\mathrm{k}}_{\mathrm{Il}}, \omega\right)$ is small. These are the natural frequencies of standing waves in the slab, which are excited by the motions of the charged particles. The fast electrons with velocities close to the phase velocity of a plasma wave are responsible for its excitation. Hence the $\mathrm{n}^{\text {th }}$ term in the emissivity expression is proportional to $F\left(\frac{\omega}{\left|\vec{k}_{n}\right|}\right)$, the number of

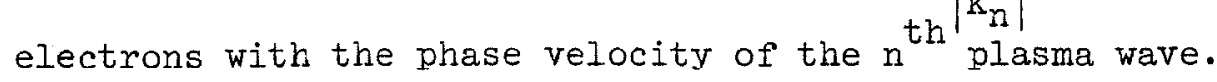

Now recall that the absorption coefficient is given in general by a very similar expression (equation 3.24), but the $n^{\text {ilt }}$ term is proportional to $F^{\prime}\left(\frac{\omega}{\left|\vec{k}_{n}\right|}\right)$, the derivative of the one-dimensional velocity distribution function. This is because the absorption is due 
to Landau damping. In a thermal equilibrium plasma there is a reiation between $F$ and $F^{\prime}$. Hence, there is aiso a relation between the emissivity and the aisorption coefficient, which agrees with Kirchhoff's law. This is discussed in the next section.

Since the emissivity corresponding to a given plasma wave is proportional to the number of electrons with the same velocity, the emissivity can be enhanced if the number of such electrons is increased. The enhancement, of emission lines caused by a. non-therma] high-energy tail in the one-dimensional distribution function $F$ is discussed in the next section.

C. Enhancement of Fmission Lines in Non-Thermal Plasmas

For purposes of comparison we first discuss the case of themal equilibrium. If a plasma slab is in thermal equilibrium at temperature $\mathrm{T}_{\mathrm{e}}$, the electron velocity distribution is a Maxwellian distribution, equation 3.27 , and so

$$
F(u)=\frac{-k T e}{m u} F^{\prime}(u)
$$

Equation 4.18 for the emissivity can therefore be written as follows for the case of thermal equilibrium:

$$
J(\theta, \omega)=\frac{\omega_{p}^{2} \omega^{3} \sin ^{2} \theta\left(k T_{e}\right)}{\pi^{2} L c^{3}} \sum_{n=1}^{\infty} \frac{-F^{\prime}\left(\frac{\omega}{\left|\vec{k}_{n}\right|}\right)}{\left|I+z_{n} / \cos \theta\right|^{2}\left|\vec{k}_{n}\right|^{4}\left|\varepsilon\left(\vec{k}_{n}, \omega\right)\right|^{2}} .
$$

It is of interest to compare this result with the emissivity calculated by using Kirchhoff's law (19). Let us imagine the plasma slab to be enclosea in a large cavity, and to be in thermal equilibrium with the walls of the cavity which are at temperature $T_{e}$. The 
radiation intensity at any point in the cavity external to the slab must have the black-body value in every direction. In particular, the power (ergs/sec) in the radiation field which flows away from the slab in a direction making an angle $\theta$ with the slab normat, per unit solid angle per unit frequency interval per unit surface area, must be $I_{B B} \cos \theta$, where $I_{B B}=\frac{\omega^{2}}{8 \pi^{3} c^{2}} k T_{e}$. This is the black-body intensity in the Rayleigh-Jeans limit $(\hbar \omega \ll \kappa T e)$ for the single polarization of interest.

If we neglect incoherent scattering* this intensity is due to the radiation from the plasma, and the reflection and transmission of the black-body radiation incident on the slab:

$$
J+\left(|R|^{2}+|T|^{2}\right) I_{B B} \cos \theta=I_{B D} \cos \theta
$$

Since the absorption coefficient of the slab is $\alpha=1-|R|^{2}-|T|^{2}$, the emissivity must be

$$
J=\alpha I_{B B} \cos \theta=a \frac{\omega^{2} \cos \theta}{8 \pi^{3} c^{2}} \times T e .
$$

With the expression for $\alpha$ given by equation 3.24 , equation 4.21 becomes identical with 4.20 .

Thus the emissivity calcuiated by using the thermal equilibrium argument is the same as that obtained from the more general expression 1.18 for the emissivity in the special case where the electron velority *

*The intensity due to incoherent (Thomson) scattering is smaller than that due to the processes considered by many orders of magnitude. If it were not negligible, the detailed balance argument given here would be invalid, because the incoherent scattering of electromagnetic waves can change the angle $\theta$ and the frequency $\omega$. 
diatribution is Maxwellian. It should be clear that tlese two methods for optaining the emissivity in thermal equilibrium are essentially independent, so that the comparison provides a consistency check on the more general method, which leads to equation 4.18 .

Also, it is worth pointing out that the assumption of "thermal equilibrium at temperature $\mathrm{T}_{\mathrm{e}}$ " really involves the electron distribulion function only. The radation, at Irequencles as high as the plasma frequency, is not affected by the motion of the ions, so no assumption is necessary about the ion distribution function.

Because the emissivity $J$ is proportional to the absorption coefficient $\alpha$ in thermal equilibrium, we expect that $J$ will have a line structure as a function of frequency, under the same conditions that $\alpha$ has such a line structure. For a thin slab $\left(k_{0} L \ll 1\right)$, the emissivity is given in the vicinity of the $n^{\text {th }}$ line $\left(\omega \simeq \omega_{n}\right)$, by equation 4.21, where $\alpha=1 / 2 \alpha_{a}$, and $\alpha_{a}$ is given by equation 3.40 . The emission lines have Lorentzian shapes, provided they are narrow, and their widths are aetermined by the Landau damping and the radiation damping of the respective plasma wave modes. It should be emphasized that these are not modes of the macroscopic charge density of the plasma, but of the microscopic density fluctuations which are responsible for the noise radiation. One can speak of the same dispersion relation and damping mechanisms as for macroscopic waves, however.

If the radiation damping is matched to the Landau damping for the $\mathrm{n}^{\text {th }}$ emission line, then the emissivity from a thermal equilibrium plasma is $1 / 2$ of the black-body value at the center of the line. 
[Recall from Section III.C that the maximum value of the absorption coefficient $\alpha$ for a thin slab is 1/2.] Otherwise, the emissivity is less than this value. There is no such restriction on the emissivity of a nonthermal plasma. It is therefore of interest to investigate the properties of the emission lines from nonthermal plasmas.

The emission lines can be discussed within the framework of the discussion of absorption lines in Section III.C. The emissivity formula 4.18 can be written in a form which is formally similar to the thermal equilibrium expression 4.20 by using tine following relation:

$$
F(u)=\frac{-\theta(u)}{m u} F^{\prime}(u)
$$

which defines the function $\theta(u)$. If the distribution function is Maxwellian, then $\theta(u)=k^{T}$, the temperature of the electrons (in energy units), and is independent of $u$. If the distribution function is not Maxwellian, then $\theta(u)$ is a function of $u$, and is not a temperature. It can be thought of as a "pseudo-temperature" which characterizes the distrihution function $F(u)$. It gives the temperature for that Maxwellian distribution which would have the same logarithmic derivative at that value of $u$.

An emission line is obtained from the corresponding absorption line [equation 3.40 for $\alpha_{a}$ ] for a nonthermal plasma as follows:

$$
J(\omega) \simeq \frac{I}{2} \alpha_{a}(\omega) \frac{\omega^{2} \cos \theta}{8 \pi^{3} c^{2}} \theta\left(\frac{\omega}{\left|\vec{k}_{n}\right|}\right)
$$

The emiasivity depends on the vclocity distribution function $F$ and is not restricted to be less than half a black-body value (which has a 
meaning only in thermal equilibrium.)

As an example of a nonthermal distribution function, suppose $F(u)$ has the following properties:

$$
F(u)=\left\{\begin{array}{l}
\frac{1}{\pi^{1 / 2} v_{e}} e^{-u^{2} / v_{e}^{2}}, u i_{e} v_{e} \\
\frac{1}{\pi^{1 / 2} v_{e}} e^{-u^{2} / v_{e}^{2}}+C u^{-s}, u>v_{e}
\end{array}\right\}
$$

This is a Maxwellian with temperature $k \mathrm{~T}_{\mathrm{e}}=\frac{1}{2} \mathrm{mv}_{\mathrm{e}}^{2}$ for low velocities. For high velocities there is, in addition, a power-law distribution which gives the distribution function a "high-energy tail". The corresponding "pseudo-temperature" has the following properties:

$$
\theta(u)=\left\{\begin{array}{cc}
k \mathrm{~T}_{e}, & u \lesssim v_{e} \\
\frac{1}{s} m u^{2}, & u>v_{e}
\end{array}\right\}
$$

(We assumed both the magnitude and slope of the Maxwellian part to be much smaller than for the power law part of the distribution function for high velocities.)

The emission lines which correspond to modes with very large phase velocities will be enhanced because of the presence of the highenergy tail. The emissivity of the $n^{\text {th }}$ such line will be multiplied by the enhancement factor

$$
f_{n}=\theta\left(\frac{\omega}{\left|\vec{k}_{n}\right|}\right) / k T_{e}
$$

This factor is much larger than unity in the example considered above 
[with $\theta(u)$ given by equation 4.25 ] if the phase velocity is much larger than the thermal velocity $v_{c}$, and $s$ is of order unity. In this example the Landau damping is also increased by the high-energy tail. Hence, the emission line is widened and the value of radiation damping for matching to the radiation field is increased. To gain an understanding of this enhancement, it is necessary to aiscuss the mechanism of the emission.

The collective response of the plasma tends to screen out the field of a charged particle for distances greater than a Debye lengtin, if the speed of the particle is less than the mean thermal speed. For a faster particie the effective screening distance is somewhat larger than a Debye length and, in addition, collective oscillations are excited in its wake (20). This excitation of plasma waves is similar to the Cerenkov radiation caused by a fast electron in a dielectric material. The more fast electrons there are, with the phase velocity of a given plasma wave mode, the greater will be the amplitude to which this mode is excited. Since the electromagnetic radiation from this mode is simply a result of continuing the electric field fluctuations into the region outside the plasma, it is clear why the corresponding emissivity should be proportional to the expected number of electrons with the phase velocity of the plasma waves, $i . e$. the distribution function evaluated at that velocity.

Although there is a relationship between the emissivity and the absorption coefficient for a thermal equilibrium plasma corresponding to the relation between the distribution function and its derivative, there is no such relationship for a nonthermal plasma. Since the 
emissivity is determined only by the electron velocity distribution, it can be enhanced over the thermal equilibrium value by high-velocity electrons in the tail of the distribution.

D. The Effect of Collisions on "Collisionless" Absorption and Emission

We have been concerned in this investigation with plasmas which can be considered nearly collisionless. We pointed out in section III.D that some collisions are necessary to justify the use of the linearized theory of plasma waves. Therefore, the effects of collisions shoula be investigated.

Encounters between many charged particles take place simuitaneously in a plasma, because of the long-range nature of the Coulomb force. The resulting electric field fluctuations cause the trajectory of each particle to undergo an almost continuous series of small deflections. The fraction of close encounters which cause large deflections is of order $\left(e^{2} / \mathrm{kT}^{3} \mathrm{n} \equiv \Lambda^{-2}\right.$, in a thermal equilibrium plasma, and is very small for temperatures and densities of most gaseous plasmas. The parameter $\Lambda$ is about ten times the number of particles in a sphere whose radius is the Debye lengti $\lambda_{D}=\left(k T / 4 \pi n e^{2}\right)^{1 / 2}$. Assuming $\Lambda$ to be very large, the vast majority of particle encounters are distant encounters, which proauce very smali deIlections.

The result is that a particle exhibits a Brownian motion, or diffusion, in velocity space and tends to slow down because of the effective frictional forces acting on 1t. The time scale for a substantial deviation of the particle trajectory because of particle encounters is the collision time $t_{c}$, where 


$$
t_{c} \simeq \frac{\Lambda}{\omega_{p} \ln \Lambda} .
$$

Therefore, in a plasma where the parameter $\Lambda$ is large, the collision time is many plasma wave periods, so the ef'tect of collisions upon the plasma waves is small.

Particle encounters, or collisions, tend to randomize any coherent wave motions in the plasma and to transform the energy of the waves into heat. The information about the location of the energy is thus lost, and we say that the energy has been absorbed by the plasma. The collisional absorption in a fully-ionized plasma can be attributed basically to dynamical friction. Because of the electric field fluctuations and the polarization force exerted on a particle by its screening cloud, the particle tenas to slow down. More precisely, its expectea velocity decreases in time from a given initial value. Because the diffusion is slow, the position of a particle remains fairly close to its expected position for many plasma periods. The theory of Brownian motion (2l) inalcates that in equations 2.10 and 2.19 we should replace $x_{0}+v_{x 0}{ }^{t}$ by $x_{n}+v_{x n}\left(I-e^{-\bar{v} t}\right) / \bar{v}$, ano multiply the right-hand side of 2.19 by $e^{-\bar{v} t}$ where $\bar{v}$ is an average collision frequency. For long wavelengths the approximate effect of collisions can be obtained by multiplying $\dot{R}_{Y}$ in equation 2.32 by $e^{-\bar{v}\left(t-t^{\prime}\right)}$, which replaces $\omega$ in equation 2.35 by $\omega+i \bar{\nu}$.

For partially-ionized gases, electron-neutral collisions must also be considered. These can be treated correctly by using the Boltzmann equation. The result is again to replace $\omega$ by $\omega+i \nu_{m}$, where 
$\nu_{m}$ is the momentum-transfer coilision frequency (22). The approximate results for a fully-ionized plasma can therefore be applied to a partially ionizeâ gas by replacing $\bar{v}$ by $\bar{v}+\nu_{m}$.

When collisions are taken into account, the approximation 2.40

must be modified; assuming $\bar{v} \ll \omega$, we have

$$
\sigma_{t}\left(\vec{k}_{n}, \omega\right) \simeq \frac{\omega_{p}^{2}(i \omega+\bar{v})}{4 \pi \omega^{2}}
$$

[A more rigorous derivation of this result, and the expression for $\bar{v}$, was given by Chang (23)]. The "transverse conductivity" therefore has a real part and so the divergence-free part of the electric field can be dissipated. The corresponding modification in the surface impedance and the absorption coefficient $a$ of the slab shows that energy can be absorbed from an incident wave by this process.

The modification of the "Iongitudinal conductivity" $\sigma_{\ell}$ due to collisions introduces the collisional damping of longitudinal plasma waves. This process is important for high phase-velocity waves, when the Ianaau damping rate is small. The approximate effect is then to जतล $2 \bar{v} / w$ tin the right-hand site of equation 3.22 and to renlace the Landau damping rate $\gamma_{n}$ in equation 3.33 by $\gamma_{n}+\bar{\nu}$. The relative width $\delta_{n}$ is therefore determined by the collisional damping rate $\bar{v}$ when the Landau damping rate is very smal7. The widtins of the ahsorption and emission lines of the plasma slab, which are associated with the plasma wave modes, are increased by the effects of collisions. Also, the value of the "radiation damping width" $r_{n}$ for which the plasma is "matched" to the radiation field, is increased, since it is 
now the sum of the Landau damping width and the collisional damping width. Thece are the modifications which are introduced by collisions; the main results of the previous work remain the same for absorption or for emission in thermal equilibrium.

The emission from a nonthermal plasma cannot be discussed quite as simply. The test-particle model of Section B can be used to calculate the emission directly, without using Kirchhoff's law. To include the effects of collisions, however, the sidcways deflectione muet be included, as well as the slowing down of the mean velocity. This corresponds to acceleration of a particle because of encounters with other particles, and results in radiation. If these accelerations are included in $\delta j_{t}^{(0)}$, which can then not be neglected in equation 4.12 , the emissivity will have a contribution due to the bremsstrahlung emisston into the transverse fleld. If lite accelerations are included in $\delta \rho_{c}^{(0)}$ in equation 4.12 as well, there is emission due to collisional excitation of plasma waves.

This procedure has been carried out by Blrmingham, Dawson and Oberman for an infinite homogeneous plasma (24). They found that, although the longitudinal emission is restricted to the frequency range $\omega_{p} \leqslant \omega \leqslant 1.4 \omega_{p}$, the emissivity in this range is much larger than that for transverse waves by a factor of order $c c^{3} / v_{e}^{3}$. For higher frequencies, where longitudinal waves do not propagate readily because of Lancau damping, the usual transverse bremsstrahlung should be the dominant radiation process.

The excitation of plasma waves by electron-ion collisions may be more important than the "collisionless" excitation by fast electrons 
for very large phase velocities. Since collisional effects are mainly due to particles of average energy, their effect on the emission is substantially the same for a nonthermal velocity distribution as for a Maxwellian, with the same mean thermal energy (25). The collisional contribution to the emissivity can therefore be calculated by using Kirchhoff's law. The result is that, in addition to the distribution function $F$ in the numerator of each term of equation 4.18 , there should he a term proportional to the mean collision frequency. If the collisional excitation and damping of plasma waves are more important than the excitation by fast electrons and Landau damping, then the emissivity has the thermal equilibrium value. It can be enhanced by nonthermal electrons, however, if the latter processes are more important than the former. 


\section{SUMMARY AND CONCLUSIONS}

The effect of collective behavior on absorption and emission of electromagnetic radiation by a bounded plasma has been investigated. A boundary-value problem has been solved in order to determine the properties of the absorption coefficient and the reflectivity near the collective resonance frequencies. The statistical properties of the noise emission near these same frequencies have also been determined by reinterpreting the solution of the boundary-value problem in terms of fluctuation quantities.

The model which has been used to represent a bounded plasma is that of a plane slab with uniform electron and ion densities. The electron velocity distribution is supposed known and independent of time. The electrons are specularly reflected when they strike the boundaries of the slab, which provides the means of containing the plasma. On the short time scales which are characteristic of plasma waves, the ions are supposed motionless.

The main assumptions which have been made are as follows:

(1) The plasma is nearly collisionless: $\omega_{p} t_{c} \gg 1$

(2) The collective oscillations are only small perturbations on the particle trajectories, so linearization is valid

(3) The distribution function is isotropic: $f_{0}=f_{0}(|\vec{v}|)$

(4) The plasma is non-relativistic: $\left\langle v^{2}\right\rangle \ll c^{2}$

(5) The slab thickness is many Debye lengths: $L / \lambda_{D} \gg 1$

(6) The mean collision rate is much smaller than the Landau damping rate, but much larger than the trapping rate: $\gamma_{n} \gg \bar{v} \gg \tau_{t r}^{-1}$ 
(7) Fluctuation quantities of higher order than those kept in the dressed test particle apprnach are negligible.

The specific results which have been obtained are tine following. If a plane electromagnetic wave is incident on the plasma slab at an angle $\theta \neq 0$, and is polarized with the electric field in the plane of incidence, then it can be partially absorbed by Landau damping of the plasma waves which it excites. If the incident wave frequency is close to one of the plasma resonance frequencies, the absorption may de substantial, but only if the slab is thin compared with the electromagnetic wavelength: $k_{0} L \ll I$. The width of the absorption line is the sum of the widths due to Landau damping and radiation damping. If the radiation damping is equal to the Landau damping for one of the lines, then the piasma is matched to the radiation field in that line, and the absorption coefficient has its maximum value of $1 / 2$ at the center of that line. The half of the inciaent power in the antisymmetric part of the field is totally absorbed at that frequency, and the other half in the symmetric part of the Iield is scattered symmetricaldy to either side (if the slab is thin: $k_{0} I \ll 1$ ). The peak values of the absorption lines do not necessarily decrease monotonically with $n$. The reflectivity also has resonance lines at the same Irequencies as the absorption lines, and with the same linewidths. The peak values of these scattering resonance lines decrease monotonically with $\mathrm{n}$. When collisions are taken into account, the Landau damplng rate $\gamma_{n}$ which appears in the theoretical results for the $n^{\text {th }}$ resonance must be replaced by $\gamma_{n}+\bar{v}$, where $\bar{v}$ is the mean collision frequency. 
Furthermore, a nearly-collisionless plasma slab emits noise radiation which is polarized with the electric vector in the plane of the slab normal and the direction of propagation. The emissivity depends on the angle $\theta$ between these two directions, and goes to zero like $\sin ^{2} \theta$ for $\theta \rightarrow 0$. Emission lines occur under the same conditions as absorption lines and at the same resonance frequencies. In thermal equilibrium the emissivity is related to the absorption coefficient by Kirchhoff's law. If the plasma is also matched to the radiation field in one of the lines, then (for a thin siab) the emissivity at the center of that line is $1 / 2$ of the black-body value. The noise radiation results from continuation of the electric field fluctuations away from the plasma, as a solution of the wave equation in free space. The fluctuations arise in the plasma because of the motions of the electrons, which can be treated using the "dressed test particle" approach. Emission lines occur at those frequencies for which the fluctuations are reinforced by the collective behavior of the plasma. The emissivity in a given line is proportional to the number of electrons with velocities about equal to the phase velocity of the piasma wave which is resonant at that frequency. The emissivity can therefore be enhanced for a nonthermal plasma with many such electrons in a high-energy tail of the distribution function. The collisional excitation of plasma waves may be the dominant mechanism for very large phase velocities.

Although high-frequency fluctuations can be observed in a plasma by incoherent scattering techniques, the scattering cross section is 
very small and it may be easier to observe them using noise emission measuremenls from a bounded plasma. In general, one would expect that the properties of the radiation from any bounded plasma would be strongly influenced by the spectrum of collective wave motions in the plasma. The comparison of the above theoretical results with the results of the microwave experiments which were mentioned in the Introduction can be of a qualitative nature only. This is because of the differences velween the discharge plasmas used in the experiments and the idealizcd plasma model used in the theoretical calculation. The most important difference is that the discharge plasma is inhomogeneous, so that the electron density is nol cunslunl in the plasma. The standing wave normal modes are reflected against the density gradient and exist only in a thin region near the plasma boundary. Since this reduces the erfective wiath of the plasma, the separabiun of the resonances in frequency is considerably larger than it would be for a homogeneous plasma of the same actual width, as used in the theoretical calcuiation. Another difference is that the discharge plasmas are usually circular cylinders, wille the theory has been worked out for a plane slab. However, the experimental results and the above theoretical results have the following features in common:

(1) The frequencies of the emission lines are approximately the same as the frequencies of the corresponding scattering resonances.

(2) The wiaths of the emissiun iines and of lhe corsesponding scattering resonances are also approximately the same.

(3) The scattering maxima decrease monotonically with increasing mode number a ( $r^{*}$ with increasing frequency $\omega_{n}$ ). 
(4) The emission maxima do not necessarily decrease monotonically, depending on the size of the plasma.

For the larger of the two discharge tubes used by Kerzar and Weissglas (3), the emission maximum of the second resonance iine is the largest, while for the smaller tube it is the first (or main) resonance line which has the largest peak value. This is understandable in terms of matching of the radiation damping to the internal damping of the plasma, since the radiation damping decreases with increasing mode number $\mathrm{n}$, but increases with the size of the plasma.

The observed line widths decrease with increasing mode number similar to the behavior of the radiation damping. But the internal damping rate must be of the same order of magnitude as the radiation damping rate, in order that the plasma ine approximately matched in the first or accona line. Hence the internal damping rate must be approximately independent of mode number. If we knew that the Landau damping rate for an inhomogeneous plasma increases sharply with moaje number as it does for a homogeneous piasma, then we could conclude that the internal damping is not Landau damping, and must be collisional. A better reason for this conclusion is that the absorption and emission in lie experiments of Kerzar and Weissglad increase in proportioin to the neutral gas density. Therefore, the primary dissipative mechanism in these discharge plasmas must be electron-neutral collisions. The corresponaing radiation mechanism is probably the excilalion of plasma waves by these collisions, which is consistent with the fact that the experimental absorption and emission spectra are very similar. 
The theory of "collisionless" absorption and emission of electromagnetic waves is therefore not entirely applicable to the experiments mentioned above. The theory cannot be completely confirmed until similar experiments are carried out with nearly-collisionless plasmas. 
REFERENCES

1. A. Dattner, Phys. Rev. Letters 10, 205 (1963).

2. J. V. Parker, J. C. Nickel, R. W. Gould, Phys. Fluids I, 1489 (1964).

3. B. Kerzar, P. Weissglas, J. Appl. Phys. 36, 2479 (1965).

4. R. W. Gould, Proc. of the Seventh International Conference on Phenomena in Ionized Gases, Belgrad, 1965, Vol. II, p. 546.

5. L. D. Landau, J. Physics (USSR) 10, 25 (1946).

6. L. Spitzer, Jr., Physics of Fully Ionized Gases, Seconà Ed., Interscience Publishers, New York 1962, pp.23-26.

7. F. C. Shure, J. Nucl. Energy C6, 1 (1964).

8. G. Bekefi, Raciation Processes in Plasmas, John Wiley and Sors, Inc., New York 1966, pp.37-4l.

9. T. H. Stix, The Theory of Plasma Waves, McGraw-Hill Book Company, New York 1962 .

10. D. C. Montgomery, D. A. Tidman, Plasma Kinetic Theory. McGraw-Hill Book Company, New York 1964, p. 52.

11. J. A. Stratton, Electromagnetic Theory, McGraw-Hill Book Company, New York 1941, p.486.

12. G.E.H. Reuter, E. H. Sondheimer, Proc. Roy. Soc. Al95, 336 (1949).

13. B. Friedman, Principles and Techniques of Applied Mathematics, John Wiley and Sons, Inc., New York 1956.

14. T. O'Neil, Phys. Fluids 8,2255 (1965).

15. A. Papoulis, Provabiliby, Random Varlables and Stochast1c Processes, McGraw-Hill Book Company, New York 1965, p.302.

16. T. H. Dupree, Phys. Fluids 6, 1714 (1963).

17. J. Hubbard, Proc. Roy. Soc. A260, 114 (1961).

18. N. Rostoker, Phys. Fluids I, 491 (1964).

19. L. D. Landau, E. M. Iifshitz, Statistical Physics, Pergamon Press Ltd., London 1958, p. 176. 
20. D. Pines, D. Bohm, Phys. Rev. 15 , 338 (1952).

21. S. Chandrasekhar, Rev. Mod. Phys. 15, I (1943).

22. I. P. Shkarofsky, T. W. Johnston, M. P. Bachynski, The Particle Kinetics of Plasmas, Addison-Wesley Publishing Company, Reading, Mass., 1966.

23. 'D. B. Chang, Phys. Fluids 5, 1558 (1962).

24. T. Birmingham, J. Dawson, C. Oberman, Phys. Fluids $\underline{8}, 297$ (1965).

25. F. Perkins, E. E. Salpeter, Phys. Rev. 139, A55 (1965). 